\title{
Energetics of internal solitary waves in a background sheared current
}

\author{
K. G. Lamb \\ Department of Applied Mathematics, University of Waterloo, Waterloo, Ontario, N2L 3G1, Canada \\ Received: 30 April 2010 - Revised: 5 August 2010 - Accepted: 14 September 2010 - Published: 8 October 2010
}

\begin{abstract}
The energetics of internal waves in the presence of a background sheared current is explored via numerical simulations for four different situations based on oceanographic conditions: the nonlinear interaction of two internal solitary waves; an internal solitary wave shoaling through a turning point; internal solitary wave reflection from a sloping boundary and a deep-water internal seiche trapped in a deep basin. In the simulations with variable water depth using the Boussinesq approximation the combination of a background sheared current, bathymetry and a rigid lid results in a change in the total energy of the system due to the work done by a pressure change that is established across the domain. A final simulation of the deep-water internal seiche in which the Boussinesq approximation is not invoked and a diffuse airwater interface is added to the system results in the energy remaining constant because the generation of surface waves prevents the establishment of a net pressure increase across the domain. The difference in the perturbation energy in the Boussinesq and non-Boussinesq simulations is accounted for by the surface waves.
\end{abstract}

\section{Introduction}

The fate of energy associated with large amplitude internal solitary-like waves (ISWs) in the ocean is an important problem that has received considerable attention primarily because of its implications for mixing. For example Sandstrom and Elliott (1984) concluded that dissipation of ISWs constitutes the primary mixing mechanism inshore of the Scotian Shelf break while Jeans and Sherwin (2001) concluded that ISWs on the Portuguese Shelf provide an important energy source for mixing. The energetics of ISWs have been studied in many localities, including the Monterey Bay and Ore-

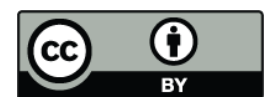

Correspondence to: K. G. Lamb (kglamb@uwaterloo.ca) gon shelves (Carter et al., 2005; Moum et al., 2007), in the St. Lawrence Estuary (Bourgault et al., 2007), Massachusetts Bay (Scotti et al., 2006) and in the South China Sea (Klymak et al., 2006) as well as in laboratory studies (Helfrich, 1992; Michallet and Ivey, 1999). Recent theoretical and numerical studies have proposed the use of an available potential energy (APE) density to calculate ISW energies (Scotti et al., 2006; Lamb, 2007, 2008; Lamb and Nguyen, 2009).

In this paper we investigate the energetics of ISWs propagating in the presence of a vertically-sheared background current. While many authors have investigated the vertical propagation of internal waves through a vertically varying background current, relatively little attention has been focussed on ISWs. Weakly nonlinear models of KdV type have been derived for stratified fluids with background sheared currents (Benney, 1966; Gear and Grimshaw, 1983). Zhou and Grimshaw (1989) extended these results to obtain evolution equations for weakly-nonlinear ISWs propagating through a slowly varying background state, including variations of the stratification, currents and water depth. Higherorder KdV-type models for ISWs in a non-Boussinesq stratified shear flow with a free surface have also been investigated (Grimshaw et al., 2002). The properties of exact ISWs in the presence of background currents were considered by Stastna and Lamb (2002). None of these authors considered wave energies, which is the focus of this paper.

In a turbulent flow or in the study of slowly varying wave trains, a background flow is normally defined using some type of averaging. In studies of turbulence an ensemble average is usually used in theoretical formulations, however in field observations or laboratory experiments it is necessary to use a spatial or temporal average. A similar approach can be used when studying slowly varying wave trains (Bretherton and Garrett, 1968; Bretherton, 1969; Whitham, 1974; Grimshaw, 1985; Craik, 1985). If the wave train varies slowly in space one may choose to define an averaging operator over several wave lengths. In the cases of turbulence and slowly varying wave trains the result is that the velocity

Published by Copernicus Publications on behalf of the European Geosciences Union and the American Geophysical Union. 
field is split into a mean and perturbation via

$\boldsymbol{u}=\langle\boldsymbol{u}\rangle+\boldsymbol{u}^{\prime}=\boldsymbol{U}+\boldsymbol{u}^{\prime}$,

where $\langle\cdot\rangle$ denotes the averaging operator. The averaging operator has the property that $\left\langle U_{i} u_{j}^{\prime}\right\rangle=U_{i}\left\langle u_{j}^{\prime}\right\rangle=0$ since $\left\langle u_{i}^{\prime}\right\rangle=0$. Here the subscripts denote components of the velocity vector. The consequence of the use of this type of averaging is that the mean kinetic energy per unit mass (the Boussinesq approximation is used in this discussion) is

$\left\langle\frac{1}{2} u_{i} u_{i}\right\rangle=\frac{1}{2} U_{i} U_{i}+\left\langle\frac{1}{2} u_{i}^{\prime} u_{i}^{\prime}\right\rangle$

where repeated indices denote summation over the velocity components. The first term on the right is the kinetic energy density of the mean flow while the second term is the mean perturbation kinetic energy density ("wave" or "turbulent" kinetic energy in the context of slowly varying waves or turbulence, respectively). Both terms are positive definite. Strictly speaking, for slowly varying wave trains $\boldsymbol{U}$ varies on slow length and time scales in which case $\left\langle U_{i} u_{j}^{\prime}\right\rangle$ may not be precisely zero. Such terms make higher-order contributions to the kinetic energy than the terms retained above.

In the context of internal solitary waves one could likewise define a background flow via averaging. For example for a two-dimensional flow a background horizontal current $U(z, t)$ could be defined as

$U(z, t)=\frac{1}{L} \int_{x_{1}}^{x_{\mathrm{r}}} u\left(x^{\prime}, z, t\right) d x^{\prime}$

where $L=x_{\mathrm{r}}-x_{1}$ is the length of the domain of interest. Then the contribution to the kinetic energy density per unit mass from the horizontal velocity component would be

$\frac{1}{2} U^{2}+U u^{\prime}+\frac{1}{2} u^{\prime 2}$

Integrating over the domain of interest the integrated kinetic energy per unit mass is

$\frac{L}{2} \int_{-H}^{0} U^{2} d z+\int_{x_{1}}^{x_{\mathrm{r}}} \int_{-H}^{0} \frac{1}{2}\left(u^{\prime 2}+w^{2}\right) d z d x$,

where the fluid is assumed to lie between $z=-H$ and $z=0$, because

$\int_{x_{1}}^{x_{\mathrm{r}}} U u^{\prime} d x=U \int_{x_{1}}^{x_{\mathrm{r}}} u^{\prime} d x=0$,

by definition. Here $w$ is the vertical velocity with zero mean. With this formulation the integrated kinetic energy has two parts, a "background" kinetic energy and a "perturbation" kinetic energy, both of which are positive definite.

In the context of internal solitary waves the definition of a background current via horizontal averaging is problematic because it depends on the length of the domain and it depends on time (e.g., it will change as two internal solitary waves interact). In this paper we use the far field state to define the background velocity field $\bar{U}(z)$ which in the cases considered here is independent of time. The horizontal integral of the horizontal velocity perturbation is no longer zero and the perturbation kinetic energy includes a term $\bar{U} u^{\prime}$ which may be negative. Because it is first-order in the perturbation velocity $u^{\prime}$ it can dominate the second-order term $u^{\prime} u^{\prime} / 2$ with the result that the contribution to the perturbation kinetic energy can be negative if $\left|\bar{U}+u^{\prime}\right|<|\bar{U}|$. This paper explores the implications of using the far-field state as the background velocity.

The numerical model used in this study can be run with and without making the Boussinesq approximation. It is described in Sect. 2. Evolution equations for the perturbation mechanical energies are derived in Sect. 3. When the Boussinesq approximation is made the perturbation kinetic energy can be split into two-terms, these being first- and secondorder in the perturbation velocity fields. Both the total perturbation energy and the second-order perturbation energies are considered. This is done because the first-order term is often not present because of the way the background velocity field is defined. While it is necessary to use the full perturbation energy, consideration of both terms illustrates the significance of the first-order energy term. It is shown that the total perturbation energy is conserved in a flat-bottomed domain, however in a domain with variable depth a pressure drop can be formed across the domain which results in net work being done on the fluid within the domain of interest and a concomitant change in energy. When the Boussinesq approximation is not made the decomposition of the perturbation energy into terms of different orders is more complicated and in this situation we consider the total perturbation energy only. The dependence of ISW energies on the strength of the background current is discussed in Sect. 4. Hyperbolic tangent density and velocity profiles are used for four sets of cases with differing depths of the pycnocline and shear layer. In Sect. 5 we turn to time-evolving wave fields. Results from four cases are presented. For these simulations the Boussinesq approximation is used and the model uses a rigid lid at the water surface. The cases are based on oceanographic conditions however the parameters are typical of laboratory experiments. The first three cases involving internal solitary waves: the nonlinear interaction of two ISWs; an ISW passing through a turning point as it shoals onto a shelf; and ISW reflection from a sloping boundary. The interaction of two ISWs is considered because it is a wave field undergoing significant evolution in a flat-bottomed domain for which the total pertubation energy is conserved. In contrast, in the other cases the total energy changes because of the variable water depth. The fourth case considered is a deep-water internal seiche trapped in a bottom basin. The mechanism behind the energy change is discussed in Sect. 6. Results from a single run of the deep-water seiche case which relaxes the Boussinesq approximation, making it possible to add a diffuse free 
surface, are presented in Sect. 7. Conclusions are presented in Sect. 8.

\section{Numerical model}

The Internal Gravity Wave (IGW) model is the twodimensional, non-hydrostatic, nonlinear model used in this work. It was first introduced in Lamb (1994) to investigate the generation and evolution of internal gravity waves in the ocean and has been extended to solve the non-Boussinesq equations. The governing equations we consider are the incompressible Navier-Stokes equations

$$
\begin{aligned}
\tilde{\rho}\left(\boldsymbol{u}_{t}+\boldsymbol{u} \cdot \nabla \boldsymbol{u}\right) & =-\nabla \tilde{p}-\tilde{\rho} g \hat{k}+\mu \nabla^{2} \boldsymbol{u}, \\
\tilde{\rho}_{t}+\boldsymbol{u} \cdot \nabla \tilde{\rho} & =\kappa \nabla^{2} \tilde{\rho}, \\
\nabla \cdot \boldsymbol{u} & =0 .
\end{aligned}
$$

Here $\boldsymbol{u}=(u, w)$ is the velocity in the vertical $x z$-plane, $\tilde{\rho}$ is the density, $\tilde{p}$ is the pressure, $\mu$ the viscosity and $\kappa$ the diffusivity. The equations are solved by first splitting the density and pressure into two parts via

$\tilde{\rho}=\rho_{0}(1+\rho)$

$\tilde{p}=-\rho_{0} g z+\rho_{0} p$,

where $\rho_{0}$ is a reference density. In the following the scaled non-dimensional density $\rho$ will be referred to simply as the density. The governing equations become

$$
\begin{aligned}
(1+\epsilon \rho)\left(\boldsymbol{u}_{t}+\boldsymbol{u} \cdot \nabla \boldsymbol{u}\right) & =-\nabla p-\rho g \hat{k}+v \nabla^{2} \boldsymbol{u}, \\
\rho_{t}+\boldsymbol{u} \cdot \nabla \rho & =\kappa \nabla^{2} \rho, \\
\nabla \cdot \boldsymbol{u} & =0 .
\end{aligned}
$$

The parameter $\epsilon$ which has been introduced is set to zero if the Boussinesq approximation is made and is set to one otherwise. $v=\mu / \rho_{0}$ is the kinematic viscosity.

The equations are solved using a second-order projection method (Bell et al., 1989; Bell and Marcus, 1992; Lamb, 1994) on a domain

$\mathcal{D}=\left\{(x, z) \| x_{l} \leq x \leq x_{\mathrm{r}},-H(x)=-H+h(x) \leq z \leq z_{\text {top }}\right\}$,

There is no normal flow through the upper and the lower boundaries. At the left boundary the inflow is specified while a radiation condition is applied at the right boundary. For these simulations there is a steady background current $\bar{U}(z)$ which is confined to a surface layer of thickness $h_{\mathrm{s}}$. The background current does not interact with the bathymetry. The domain is sufficiently long that no perturbations reach the boundary. The numerical model uses a quadrilateral grid constructed using vertically stretched terrain-following coordinates.

Some of the model simulations which use the Boussinesq approximation are initialized with exact ISWs. These waves are calculated by solving the Dubreil-Jacotin-Long (DJL) equation, extended to include background currents (Stastna and Lamb, 2002; Lamb, 2003), to find the vertical displacement, $\eta(x, z)$ of streamlines passing through $(x, z)$ relative to its far-upstream height in a reference frame moving with the wave. The DJL equation is

$$
\begin{aligned}
& \nabla^{2} \eta+\frac{\bar{U}^{\prime}(z-\eta)}{c-\bar{U}(z-\eta)}\left(\eta_{x}^{2}+\left(1-\eta_{z}\right)^{2}-1\right) \\
& +\frac{N^{2}(z-\eta)}{(c-\bar{U}(c-\eta))^{2}} \eta=0,
\end{aligned}
$$

which is solved with boundary conditions $\eta=0$ at $z=0,-H$ and $\eta=0$ at the lateral boundaries of the subdomain in which the waves are computed. For a sufficiently wide subdomain, as used here, these are equivalent to $\eta=0$ as $x \rightarrow \pm \infty$, as appropriate for solitary waves. The propagation speed of the solitary wave relative to the background flow $\bar{U}(z), c$, is an eigenvalue which is found as part of the solution. The DJL equation is solved using an iterative method based on a variational formulation of the problem (Turkington et al., 1991; Stastna and Lamb, 2002). This method yields an ISW with a specified available potential energy.

\section{Energy conservation and energy flux}

Neglecting viscous and diffusive effects the pseudo-energy equation is

$\frac{\partial}{\partial t}\left(E_{\mathrm{k}}+E_{\mathrm{a}}\right)+\nabla \cdot\left(\boldsymbol{u}\left(E_{\mathrm{k}}+E_{\mathrm{a}}+p_{\mathrm{d}}\right)\right)=0$,

where $p_{\mathrm{d}}$ is the pressure disturbance relative to the hydrostatic pressure of the undisturbed flow $\bar{p}(z)$,

$E_{\mathrm{k}}=\frac{1}{2} \rho_{0}(1+\epsilon \rho)\left(u^{2}+w^{2}\right)$,

is the kinetic energy density and

$E_{\mathrm{a}}(x, z, t)=\rho_{0} g \int_{z}^{z^{*}(x, z, t)}(\bar{\rho}(s)-\rho(x, z, t)) d s$,

is the available potential energy density. Here $\bar{\rho}(z)$ is the reference density and $z^{*}(x, z, t)$ is the height of the fluid particle at $(x, z, t)$ in the reference stratification (Scotti et al., 2006; Lamb, 2007, 2008; Lamb and Nguyen, 2009). In the following we use the background stratification as the reference density which is appropriate for calculating the available potential energy in an infinitely long domain Lamb (2008).

All the cases considered here involve a steady background current $\bar{U}$. Let

$u=\bar{U}(z)+u^{\prime}$

where $u^{\prime}$ is the horizontal velocity perturbation. 


\subsection{Energy equations under the Boussinesq approximation}

If the Boussinesq approximation is made the kinetic energy density (per unit volume) can be split into three terms

$E_{\mathrm{k}}=E_{\mathrm{k} 0}+E_{\mathrm{k} 1}+E_{\mathrm{k} 2}$,

where

$$
\begin{aligned}
& E_{\mathrm{k} 0}=\frac{\rho_{0}}{2} \bar{U}^{2}, \\
& E_{\mathrm{k} 1}=\rho_{0} \bar{U} u^{\prime}, \\
& E_{\mathrm{k} 2}=\frac{\rho_{0}}{2}\left(u^{\prime 2}+w^{2}\right),
\end{aligned}
$$

are the contributions to the kinetic energy density which are of order zero, one and two in the perturbation velocities. We will refer to

$E_{\mathrm{kp}}=E_{\mathrm{k} 1}+E_{\mathrm{k} 2}$,

as the perturbation kinetic energy density.

Since $\bar{U}$ is independent of time $\frac{\partial}{\partial t}\left(E_{\mathrm{k}}\right)=\frac{\partial}{\partial t}\left(E_{\mathrm{k} 1}+E_{\mathrm{k} 2}\right)$. Now

$$
\begin{aligned}
\frac{\partial}{\partial t} E_{\mathrm{k} 1} & =\rho_{0} \bar{U} u_{t}^{\prime}, \\
& =-\rho_{0} \bar{U} \nabla \cdot(u \boldsymbol{u})-\bar{U} \nabla \cdot\left(p_{\mathrm{d}}, 0\right), \\
& =-\nabla \cdot\left(\rho_{0} u \bar{U} \boldsymbol{u}+\left(p_{d} \bar{U}, 0\right)\right)+\rho_{0} u w \frac{d \bar{U}}{d z} .
\end{aligned}
$$

Rewriting the last term using

$$
\begin{aligned}
u w \frac{d \bar{U}}{d z} & =u^{\prime} w \frac{d \bar{U}}{d z}+\bar{U} w \frac{d \bar{U}}{d z}, \\
& =u^{\prime} w \frac{d \bar{U}}{d z}+\nabla \cdot\left(\frac{1}{2} \bar{U}^{2} \boldsymbol{u}\right),
\end{aligned}
$$

allows us to write the evolution equation for $E_{\mathrm{k} 1}$ as

$$
\frac{\partial}{\partial t} E_{\mathrm{k} 1}+\nabla \cdot\left(\boldsymbol{u}\left(E_{\mathrm{k} 0}+E_{\mathrm{k} 1}\right)+\left(p_{\mathrm{d}} \bar{U}, 0\right)\right)-u^{\prime} w \frac{d \bar{U}}{d z}=0 .
$$

Subtracting this from (12) results in

$$
\begin{aligned}
& \frac{\partial}{\partial t}\left(E_{\mathrm{k} 2}+E_{\mathrm{a}}\right)+\nabla \cdot\left(\boldsymbol{u}\left(E_{\mathrm{k} 2}+E_{\mathrm{a}}\right)+p_{\mathrm{d}}\left(u^{\prime}, w\right)\right) \\
& +\rho_{0} u^{\prime} w \frac{d \bar{U}}{d z}=0 .
\end{aligned}
$$

This equation says that the second-order perturbation energy is not conserved: it can change due to the shear production term $u^{\prime} w \frac{d \bar{U}}{d z}$ which is familiar from turbulence theory. It acts to exchange energy between the first- and second-order energy terms. Note however that the shear production term is non-zero only in regions where $\frac{d \bar{U}}{d z}$ is non-zero, i.e., at the depth of the shear layer in the background flow which does not necessarily coincide with the depth of the perturbed shear layer. The reason is that $u^{\prime}$ is the horizontal velocity perturbation relative to the background flow $\bar{U}(z)$. When a fluid particle moves vertically, with constant velocity $\boldsymbol{u}$, through a depth where the background flow has strong shear, the kinetic energy of the fluid particle may not change but as $\bar{U}$ changes so does $u^{\prime}$ and there can be large changes in $E_{\mathrm{k} 2}$.

In the following we will use bars over the various energy densities to indicate values integrated over the domain $\mathcal{D}$. In the simulations initialized with ISWs considered below the initial wave energies are based on integrals over a subdomain containing a single ISW.

Integrating (12) over $\mathcal{D}$ gives the energy balance equation

$\frac{d}{d t} \bar{E}_{\mathrm{pseudo}}=\left.\left(K_{\mathrm{f}}+\mathrm{APE}_{\mathrm{f}}+W\right)\right|_{x_{\mathrm{r}}} ^{x_{\ell}}$,

where $E_{\mathrm{pseudo}}=E_{\mathrm{a}}+E_{\mathrm{k}}$ is the total pseudo-energy density,

$$
\begin{aligned}
K_{\mathrm{f}} & =\int_{-H(x)}^{0} u E_{\mathrm{k}} d z, \\
\mathrm{APE}_{\mathrm{f}} & =\int_{-H(x)}^{0} u E_{\mathrm{a}} d z,
\end{aligned}
$$

are the vertically integrated kinetic and available potential energy flux densities, and

$$
W=\int_{-H(x)}^{0} u p_{\mathrm{d}} d z
$$

is the rate work is done by the pressure perturbation. The total energy flux through a horizontal location $x$ is $E_{\mathrm{f}}=K_{\mathrm{f}}+$ $\mathrm{APE}_{\mathrm{f}}+W$.

By definition $\bar{E}_{\mathrm{k} 0}$ is constant in time so

$\frac{d}{d t}\left(\bar{E}_{\mathrm{kp}}+\bar{E}_{\mathrm{a}}\right)=\left.\left(K_{\mathrm{f}}+\mathrm{APE}_{\mathrm{f}}+W\right)\right|_{x_{\mathrm{r}}} ^{x_{\ell}}$,

To further simplify the energy equation we assume that no waves arrive at the boundaries in the time of interest. In particular we will assume that $u^{\prime}=w=0$ at the lateral boundaries. As we will see below we will have to allow for a change in the pressure $p_{\mathrm{d}}$. Thus at $x=x_{\mathrm{l}}$ and $x_{\mathrm{r}}, \mathrm{APE}_{\mathrm{f}}=0$ and

$K_{\mathrm{f}}=\int_{-H(x)}^{0} \frac{\rho_{0}}{2} \bar{U}^{3}(z) d z$.

This integral has the same value at the two lateral boundaries, hence

$\frac{d}{d t}\left(\bar{E}_{\mathrm{kp}}+\bar{E}_{\mathrm{a}}\right)=\left.\int_{-H}^{0} \bar{U}(z) p_{\mathrm{d}}\right|_{x_{\mathrm{r}}} ^{x_{\ell}} d z$.

At the boundaries the pressure is in hydrostatic balance with the undisturbed density field $\bar{\rho}(z)$ and hence is given by

$p=p_{\mathrm{s}}+\int_{z}^{0} \rho_{0} g \bar{\rho}(z) d z$, 
where $p_{\mathrm{s}}$ is the surface pressure, so

$\frac{d}{d t}\left(\bar{E}_{\mathrm{kp}}+\bar{E}_{\mathrm{a}}\right)=-\Delta p_{\mathrm{s}} M$,

where

$M=\int_{-H}^{0} \bar{U}(z) d z$

is the volume flux associated with the background current entering through the left boundary and $\Delta p_{\mathrm{s}}=p_{\mathrm{s}}\left(x_{\mathrm{r}}\right)-p_{\mathrm{s}}\left(x_{1}\right)$ is the change in the surface pressure across the domain.

Since the volume flux

$\int_{-H(x)}^{0} u d z$

is independent of $x$ and $t$

$$
\begin{aligned}
0=\int_{-H(x)}^{0} \rho_{0} u_{t} d z & =-\int_{-H(x)}^{0} \nabla \cdot\left(\rho_{0} u \boldsymbol{u}+\left(p_{\mathrm{d}}, 0\right)\right) d z, \\
& =-\oint\left(p_{\mathrm{d}}, 0\right) \cdot \hat{n} d s,
\end{aligned}
$$

since $\boldsymbol{u} \cdot \hat{n}=0$ along the lower and upper boundaries and the integrals of $\boldsymbol{u} \cdot \hat{n}$ along the left and right lateral boundaries, where $u=\bar{U}$, cancel.

If the water depth $H$ is constant this gives

$$
\begin{aligned}
0 & =-\left.\int_{-H}^{0} p_{\mathrm{d}}\right|_{x_{1}} ^{x_{\mathrm{r}}} d z, \\
& =-\left.\int_{-H}^{0} \Delta p_{\mathrm{s}}\right|_{x_{1}} ^{x_{\mathrm{r}}} d z, \\
& =-\Delta p_{\mathrm{s}} H .
\end{aligned}
$$

Hence, if the depth is uniform the change in the surface pressure across the domain is zero and (30) reduces to

$$
\frac{d}{d t}\left(\bar{E}_{\mathrm{kp}}+\bar{E}_{\mathrm{a}}\right)=0 .
$$

If the water depth is not uniform $\Delta p_{\mathrm{s}}$ is not necessarily zero and the perturbation energy $\bar{E}_{\mathrm{kp}}+\bar{E}_{\mathrm{a}}$ is not necessarily constant in time.

Integrating the evolution for $E_{\mathrm{k} 1}$ gives

$$
\begin{aligned}
& \frac{d \bar{E}_{\mathrm{k} 1}}{d t}=-\oint \rho_{0} \bar{U} u^{\prime} \boldsymbol{u} \cdot \hat{n} d s-\oint \bar{U}\left(p_{\mathrm{d}}, 0\right) \cdot \hat{n} d s \\
& +\iint_{\mathcal{D}} \rho_{0} u^{\prime} w \bar{U}^{\prime} d z d x .
\end{aligned}
$$

The first term on the right is zero because either $u^{\prime}$ or $\boldsymbol{u} \cdot \hat{n}$ are zero on the domain boundary. The second term is also zero along the upper and lower boundaries since the background current is assumed to be confined to a region above the bottom. Thus

$$
\frac{d \bar{E}_{\mathrm{k} 1}}{d t}=-\Delta p_{\mathrm{s}} M+P
$$

where

$$
P=\iint_{\mathcal{D}} \rho_{0} u^{\prime} w \frac{d \bar{U}}{d z} d z d x
$$

is the (integrated) shear production term.

Subtracting this from (30) gives an evolution equation for the second-order energy perturbation

$\frac{d}{d t}\left(\bar{E}_{\mathrm{k} 2}+\bar{E}_{\mathrm{a}}\right)=-P$.

In this equation each term is second-order in the perturbation.

\subsection{Energy equations without the Boussinesq approximation}

If the Boussinesq approximation is not made then it is less convenient to separate the kinetic energy into terms of different order in the perturbation quantities as there are many more terms and there is in addition a third-order term. Thus, if the Boussinesq approximation is not made we only consider the perturbation kinetic energy density which now has the form

$E_{\mathrm{kp}}=E_{\mathrm{k}}-\frac{\rho_{0}(1+\bar{\rho})}{2} \bar{U}^{2}$.

The integrated energy equation (30) is still satisfied.

\section{Energetics of internal solitary waves under the Boussinesq approximation}

Before considering evolving internal wave fields some results on the energetics of internal solitary waves are presented. Further properties for linear and hyperbolic tangent background currents can be found in Stastna and Lamb (2002).

We consider background stratification and velocity fields of the form

$\rho=\bar{\rho}(z)=-\frac{\Delta \rho}{2} \tanh \left(\frac{z-z_{\mathrm{pyc}}}{d_{\mathrm{pyc}}}\right)$,

and

$\bar{U}(z)=\frac{U_{m}}{2}\left(1+\tanh \left(\frac{z-z_{\mathrm{s}}}{d_{\mathrm{s}}}\right)\right)$.

and focus on wave energies for cases with waves propagating against a surface current, that is, for $U_{\mathrm{m}}<0$ and wave propagation speeds $c>0$. A water depth of $H=1 \mathrm{~m}$ is used and the reference density is taken as $\rho_{0}=1000 \mathrm{~kg} \mathrm{~m}^{-3}$.

In Fig. 1 the integrated available potential energy $\bar{E}_{\mathrm{a}}$ and perturbation kinetic energies $\bar{E}_{\mathrm{kp}}$ are plotted as a function of wave amplitude $\max |\eta|$ for four sets of cases. They all use a density jump of $\Delta \rho=0.04$ across the pycnocline and the thickness of the pycnocline and shear layer are fixed at $d_{\text {pyc }}=0.05 \mathrm{~m}$ and $d_{\mathrm{s}}=0.03 \mathrm{~m}$, respectively. Note that scaling $\Delta \rho$ and $U_{m}$ by factors of $r$ and $\sqrt{r}$ respectively yields 

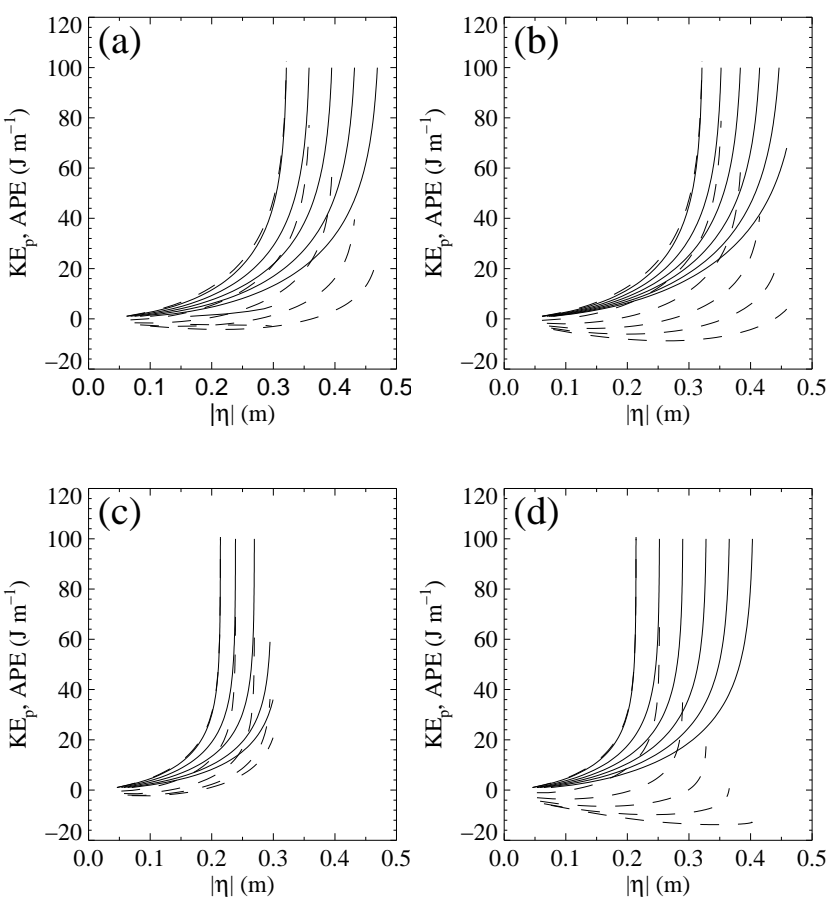

Fig. 1. $\bar{E}_{\mathrm{kp}}$ (dashed) and $\bar{E}_{\mathrm{a}}$ as a function of wave amplitude using hyperbolic tangent stratifications with $\Delta \rho=0.04, d_{\text {pyc }}=$ $0.05 \mathrm{~m}$ and hyperbolic tangent shear layers with $d_{s}=0.03 \mathrm{~m}$. (a) Coincident pycnocline and shear layer near the surface with $\left(z_{\mathrm{s}}, z_{\text {pyc }}\right)=(-0.2,-0.2) \mathrm{m}$. (b) Shear layer below the pycnocline with $\left(z_{\mathrm{s}}, z_{\text {pyc }}\right)=(-0.3,-0.2) \mathrm{m}$. (c) Shear layer above the pycnocline with $\left(z_{\mathrm{s}}, z_{\text {pyc }}\right)=(-0.2,-0.3) \mathrm{m}$. (d) Coincident pycnocline and shear layer near the mid-depth with $\left(z_{\mathrm{s}}, z_{\text {pyc }}\right)=$ $(-0.3,-0.3) \mathrm{m}$. In each plot the sets of curves are for different values of $U_{m}$ starting at $U_{m}=0$ (left most curve) and decreasing by increments of $-0.05 \mathrm{~m} \mathrm{~s}^{-1}$. Total water depth is $H=1 \mathrm{~m}$.

a wave with the same shape (i.e., $\eta(x, z)$ is unchanged) with $c, u^{\prime}$ and $w$ increased by a factor of $\sqrt{r}$ and $\bar{E}_{\mathrm{a}}$ and $\bar{E}_{\mathrm{kp}}$ increased by a factor of $r$. The density change corresponds to upper and lower layer densities of 1000 and $1040 \mathrm{~kg} \mathrm{~m}^{-3}$, typical of many laboratory experiments, using a reference density of $\rho_{0}=1000 \mathrm{~kg} \mathrm{~m}^{-3}$. Waves were calculated for a range of available potential energies with $\bar{E}_{\mathrm{a}}$ varying from 1 to $100 \mathrm{~J} \mathrm{~m}^{-1}$ in increments of $1 \mathrm{~J} \mathrm{~m}^{-1}$ using a $\mathrm{KdV}$ internal solitary wave with $\bar{E}_{\mathrm{a}}=1 \mathrm{~J} \mathrm{~m}^{-1}$ as the initial guess for the iterative solver. Thereafter as $\bar{E}_{\mathrm{a}}$ is increased along each curve the previous solution is used as the initial guess.

Figure 1 shows results for four sets of values of $\left(z_{\mathrm{s}}, z_{\mathrm{pyc}}\right)$. In each set waves are calculated for different values of $U_{m}$, starting at $U_{m}=0$ and decreasing by increments of $-0.05 \mathrm{~m} \mathrm{~s}^{-1}$.

Figure 1a shows the results for a coincident shear layer and pycnocline using $\left(z_{\mathrm{s}}, z_{\text {pyc }}\right)=(-0.2,-0.2) \mathrm{m}$. The leftmost curves show the results for $U_{m}=0$. As $\bar{E}_{\mathrm{a}}$ increases the wave amplitude asymptotes to a limiting value. This is indicative of the conjugate flow limit being reached at which waves flat- ten in the centre and broaden as the energy in the waves is increased (Tung et al., 1982; Turner and Vanden-Broeck, 1988; Lamb and Wan, 1998). When there is no background current the maximum ISW amplitude is $-0.32 \mathrm{~m}$ (negative implying a wave of depression), slightly larger than the distance of the pycnocline from the mid-depth. For a two layer fluid the maximum displacement would be $-0.3 \mathrm{~m}$ but because of the relatively thick pycnocline the maximum amplitude is slightly larger. Wave propagation speeds increase from 0.253 to $0.296 \mathrm{~m} \mathrm{~s}^{-1}$ as the wave amplitude increases. The kinetic energy of the wave is slightly larger than the available potential energy (it must always be larger in the absence of a background current - see Turkington et al. (1991); Lamb and Nguyen (2009)).

The limiting amplitudes increase linearly as $U_{m}$ decreases which is consistent with theoretical predictions for conjugate flows. Conjugate flows for two- and three-layer flows were explored in Lamb (2000). There it was shown that under the Boussinesq approximation the conjugate flow amplitude for a two layer flow with coincident density and velocity jumps is

$\eta_{\text {conj }}=-z_{\text {pyc }}+0.5\left(-H+\sqrt{\frac{H}{\Delta \rho g}} U_{m}\right)$,

where $H=1 \mathrm{~m}$ is the water depth. For the cases with $z_{\mathrm{pyc}}=$ $z_{\mathrm{s}}=-0.2 \mathrm{~m}$ shown in Fig. 1a this gives

$\eta_{\text {conj }}=-0.3+0.7982 U_{m}$.

For $U_{m}=-0.2 \mathrm{~m} \mathrm{~s}^{-1}$ we have $\eta_{\text {conj }}=-0.46 \mathrm{~m}$. For the computed ISWs, the largest wave amplitude for $U_{m}=$ $-0.2 \mathrm{~m} \mathrm{~s}^{-1}$ is $-0.47 \mathrm{~m}$. Differences can be expected because the density and velocity profiles used to calculate ISWs undergo smooth transitions with a relatively broad pycnocline.

As $U_{m}$ decreases the kinetic energy decreases, becoming negative for small amplitude waves, and then positive for sufficiently large waves. The kinetic energy is always less than the available potential energy for the cases with $U_{m}<0$. The negative $\bar{E}_{\mathrm{kp}}$ values occur because the wave induced velocity is positive in the upper layer. Because it is in the opposite direction to the background flow the result is a decrease in the horizontal velocity in the upper layer, i.e., $\left|\bar{U}+u^{\prime}\right|<|\bar{U}|$, with a corresponding decrease in kinetic energy. Beneath the pycnocline, where there is no background flow, the wave induced currents are negative and act to increase the kinetic energy. The reduction in kinetic energy above the pycnocline, being first-order in the wave amplitude for small waves, is larger than the increase beneath it and the total kinetic energy in the system is reduced until $U+u^{\prime}$ becomes sufficiently large.

For $U_{m}=0$ the largest wave has a minimum Richardson number $(R i)$ in the pycnocline of 0.24 . For $U_{m}=-0.2 \mathrm{~m} \mathrm{~s}^{-1}$, the background state has a minimum $R i$ in the pycnocline of 0.35 . For small waves the minimum $R i$ initially increases 
because the wave induced shear has the opposite sign to the background shear, before decreasing as the wave amplitude continues to increase. For the largest wave computed the Richardson number in the pycnocline has two minimums: 0.4 at $z=-0.61 \mathrm{~m}$ and 0.28 at $=-0.69 \mathrm{~m}$. When $U_{m}$ is decreased to $-0.25 \mathrm{~m} \mathrm{~s}^{-1}$ the background state has a minimum Richardson number in the pycnocline of 0.23 . When $\bar{E}_{\mathrm{a}}=2 \mathrm{~J} \mathrm{~m}^{-1}$ the maximum of $u$ exceeds the wave propagation speed of $0.11 \mathrm{~m} \mathrm{~s}^{-1}$ hence there is a wave induced critical level where the horizontal velocity is equal to the wave propagation speed. Figure 2 compares the background velocity profile with the wave induced velocity profiles down the centre of the waves for $\bar{E}_{\mathrm{a}}=1$ and $2 \mathrm{~J} \mathrm{~m}^{-1}$. For the larger of these two waves there is a small density overturn in the pycnocline implying the formation of closed streamlines. Waves as large as $\bar{E}_{\mathrm{a}}=5 \mathrm{~J} \mathrm{~m}^{-1}$ were computed before the numerical method failed to converge.

When the pycnocline thickness is reduced the wave amplitudes are smaller, becoming closer to the two-layer conjugate flow values, while the integrated kinetic energy perturbation is almost unchanged. For example, for $U_{m}=-0.2 \mathrm{~m} \mathrm{~s}^{-1}$ when $d_{\text {pyc }}$ is reduced to $0.025 \mathrm{~m}$ the wave amplitude for the largest wave is reduced by $2.5 \%$ while the largest change (over the range of $\bar{E}_{\text {a values) of the integrated kinetic energy }}$ perturbation is $1 \%$ of that of the largest wave. One significant difference is that now waves for $U_{m}=-0.25 \mathrm{~m} \mathrm{~s}^{-1}$ can be computed up to $\bar{E}_{\mathrm{a}}=100 \mathrm{~J} \mathrm{~m}^{-1}$. Figure 2 includes the wave induced velocity profile down the centre of the wave with $\bar{E}_{\mathrm{a}}=1 \mathrm{~J} \mathrm{~m}^{-1}$. Compared with the corresponding case using $d_{\text {pyc }}=0.05 \mathrm{~m}$ the positive velocity maximum is no longer present. For $U_{m}=-0.3 \mathrm{~m} \mathrm{~s}^{-1}$ it reappears and waves with $\bar{E}_{\mathrm{a}}$ larger than $1 \mathrm{~J} \mathrm{~m}^{-1}$ could not be computed.

When the shear layer is moved below the pycnocline to $z_{s}=-0.3 \mathrm{~m}$, Fig. 1b, wave amplitudes are slightly smaller and $\bar{E}_{\mathrm{kp}}$ becomes significantly more negative over a wider range of wave amplitudes. For $U_{m}=-0.25 \mathrm{~m} \mathrm{~s}^{-1}$ waves could be computed up to $\bar{E}_{\mathrm{a}}=68 \mathrm{~J} \mathrm{~m}^{-1}$. The Richardson number of the background state has a minimum of 0.0135 in the shear layer and is extremely large in the pycnocline. For the largest wave computed the minimum $R i$ is 0.03 in the shear layer and 0.2 in the pycnocline. When $d_{\text {pyc }}$ is reduced to $0.01 \mathrm{~m}$ waves could be computed up to $\bar{E}_{\mathrm{a}}=100 \mathrm{~J} \mathrm{~m}^{-1}$ at which point the minimum Richardson number in the pycnocline is 0.042 while the Richardson number in the shear layer is extremely small due to the now very weak stratification across it.

For these cases $\bar{E}_{\mathrm{kp}}$ becomes significantly more negative over a wider range of wave amplitudes compared with the cases in Fig. 1a.

When the shear layer is above the pycnocline, $\left(z_{\mathrm{s}}, d_{\text {pyc }}\right)=$ $(-0.2,-0.3) \mathrm{m}$, limitations on wave amplitudes that could be calculated were encountered for $U_{m}=-0.15$ and $-0.2 \mathrm{~m} \mathrm{~s}^{-1}$ (Fig. 1c). For $U_{m}=-0.2 \mathrm{~m} \mathrm{~s}^{-1}$ the Richardson number of the background state has a minimum of 0.02 in the shear layer. The largest wave, $\bar{E}_{\mathrm{a}}=36 \mathrm{~J} \mathrm{~m}^{-1}$, has mini-

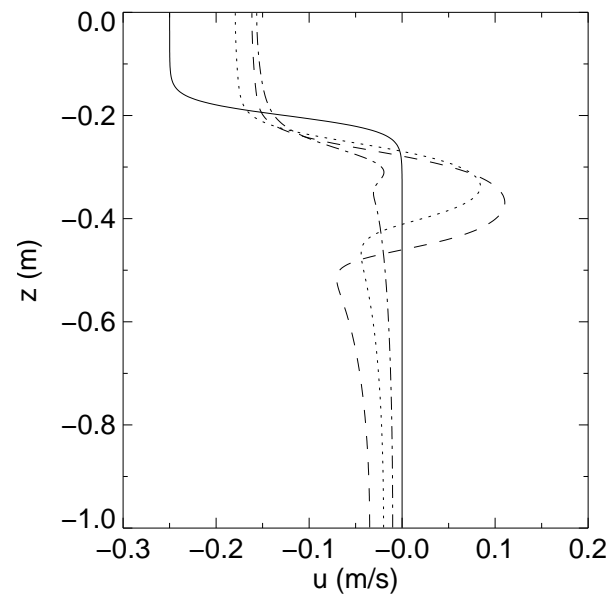

Fig. 2. Horizontal velocity profiles for waves using $\Delta \rho=0.04$, $U_{m}=-0.25 \mathrm{~m} \mathrm{~s}^{-1},\left(d_{\mathrm{s}}, z_{\mathrm{s}}\right)=(0.03,-0.2) \mathrm{m}$ and $z_{\mathrm{pyc}}=-0.2 \mathrm{~m}$. Shown is the background velocity profile (solid). The other curves are horizontal velocity profiles down the centre of an ISW. Two cases using $d_{\text {pyc }}=0.05 \mathrm{~m}$ are shown for $\bar{E}_{\mathrm{a}}=1$ (dots) and 2 (dashed) $\mathrm{J} \mathrm{m}^{-1}$. The dash-dot curve shows the velocity profile for a wave with a thinner pycnocline, $d_{\text {pyc }}=0.025 \mathrm{~m}$, with $\bar{E}_{\mathrm{a}}=$ $1 \mathrm{~J} \mathrm{~m}^{-1}$.

mum Richardson numbers of 0.015 and 0.182 in the shear layer and pycnocline, respectively. The wave amplitudes are smaller than in the previous cases because the pycnocline is closer to the mid-depth. The maximum wave amplitude is $-0.214 \mathrm{~m}$ when $U_{m}=0$.

When the shear layer and pycnocline are both centred at $z=-0.3 \mathrm{~m}$ waves were obtained for all values of $\bar{E}_{\mathrm{a}}$ and $U_{m}$. The largest negative integrated perturbation kinetic energy values are attained, and for $U_{m}=-0.25 \mathrm{~m} \mathrm{~s}^{-1}$ they are negative for the largest wave computed, with a minimum of $-13.8 \mathrm{~J} \mathrm{~m}^{-1}$.

Figure 3 shows the shape of the isopycnal undergoing maximum displacement for a series of waves with varying $U_{m}$ and $\bar{E}_{\text {a }}$ fixed to $10 \mathrm{~J} \mathrm{~m}^{-1}$. The stratifications and background currents used are the same as those in Fig. 1.

\section{Energy evolution for cases using the Boussinesq approximation}

We now turn to the results of numerical simulations. Using the Boussinesq approximation four different physical situations are considered. The first three involve internal solitary waves while the fourth is a deep-water seiche. The first two of these, the interaction of two ISWs and a shoaling ISW passing through a turning point, do not include viscous or diffusive terms. Nor does the deep seiche simulation. For the case of an ISW reflecting off a sloping bottom viscosity and diffusion are included. A deep water depth of $H=1 \mathrm{~m}$ is used with the surface at $z_{\text {top }}=0$. 

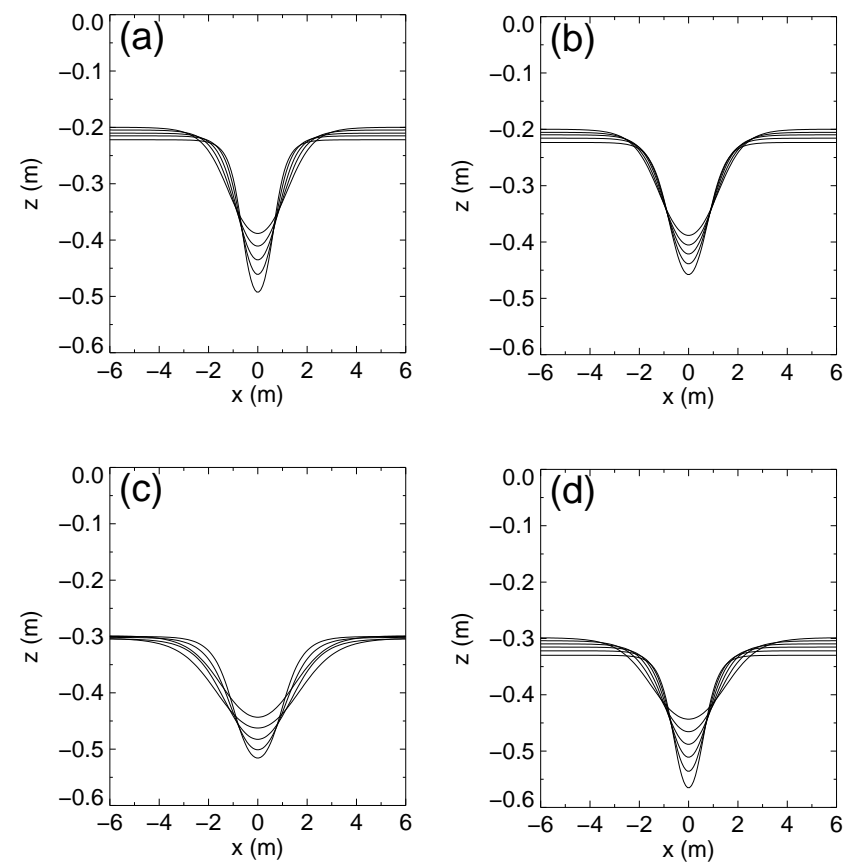

Fig. 3. Isopycnals undergoing maximum displacement for waves with $\bar{E}_{\mathrm{a}}=10 \mathrm{~J} \mathrm{~m}^{-1}$. Stratifications and currents as in Fig. 1. As $U_{m}$ decreases from 0 the waves get narrower and the maximum isopycnal displacement increases. The isopycnal undergoing maximum displacements shifts downward except for the cases in (c), for which it initially shifts downward and then upwards.

\subsection{ISW interaction}

The first example is the interaction of two ISWs of different amplitude. For this simulation the bottom is flat and as shown above the pressure drop across the domain must be zero. Hence the perturbation energy $\bar{E}_{\mathrm{kp}}+\bar{E}_{\mathrm{a}}$ is conserved. The background density and velocity profiles are given by (41) and (42) with $\Delta \rho=0.04,\left(z_{\text {pyc }}, d_{\text {pyc }}\right)=(-0.2,0.03) \mathrm{m}$, $U_{m}=-0.1 \mathrm{~m} \mathrm{~s}^{-1}$ and $\left(z_{\mathrm{s}}, d_{\mathrm{s}}\right)=(-0.3,0.03) \mathrm{m}$. These are the parameters used for cases depicted in Fig. 1b.

Results from a simulation with waves having initial available potential energies of $\bar{E}_{\mathrm{a}}=2.5$ and $80 \mathrm{~J} \mathrm{~m}^{-1}$ are shown in Fig. 4. The simulation is done in a reference frame moving with the average propagation speed of the two right-ward propagating waves. The figure shows the fluid velocity relative to the bottom layer. Initially the large wave trails the smaller wave (panel a). The initial waves have amplitudes of -0.11 and $-0.38 \mathrm{~m}$ with corresponding initial perturbation kinetic energies $\bar{E}_{\mathrm{kp}}$ of -2.0 and $46.4 \mathrm{~J} \mathrm{~m}^{-1}$. The waves at approximately the mid-point of the interaction are showed in panel $b$ while the two waves after they separate are shown in panel c.

The energetics for the interacting ISWs is shown in Fig. 5. The top panel shows the time evolution of $\bar{E}_{\mathrm{a}}, \bar{E}_{\mathrm{kp}}$ and $\bar{E}_{\mathrm{k} 2}$. To more clearly show the relative changes the variation of $\bar{E}_{\mathrm{kp}}$ and $\bar{E}_{\mathrm{a}}$ and their sum from their initial values is shown in
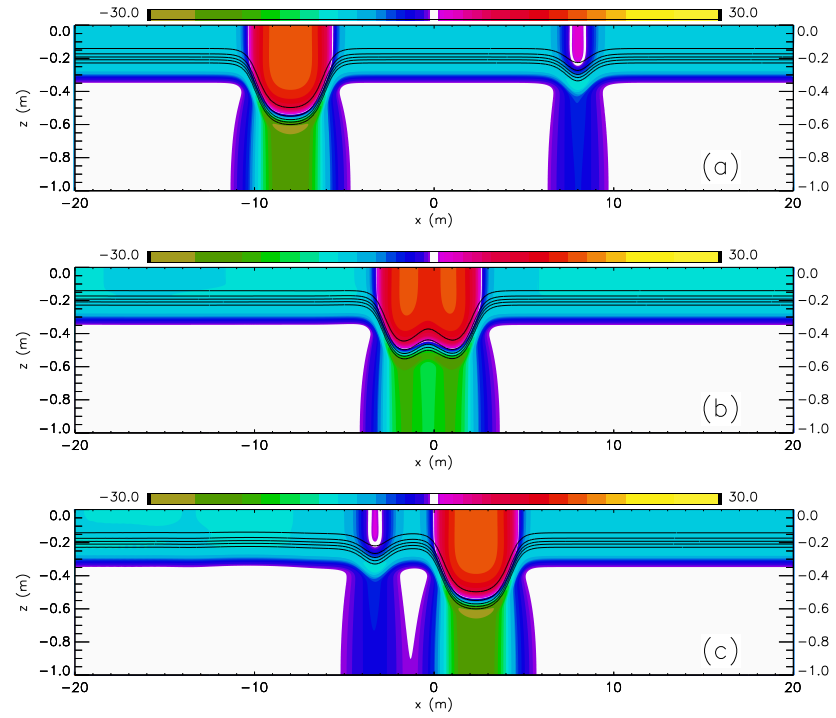

Fig. 4. Contour plots of the density field (solid lines) and horizontal velocity field (colours) for two interacting ISWs with initial APEs of 2.5 and $80 \mathrm{~J} \mathrm{~m}^{-1}$. Simulation is done in a reference frame moving with the average propagation speed of the waves. The horizontal velocity field shown is that in a reference frame fixed with the far field fluid below the surface current. (a) Initial waves. (b) Waves at middle of the interaction $(t=310 \mathrm{~s})$. (c) $t=400 \mathrm{~s}$. The white regions indicate regions where $|u|<0.0013 \mathrm{~m} \mathrm{~s}^{-1}$.
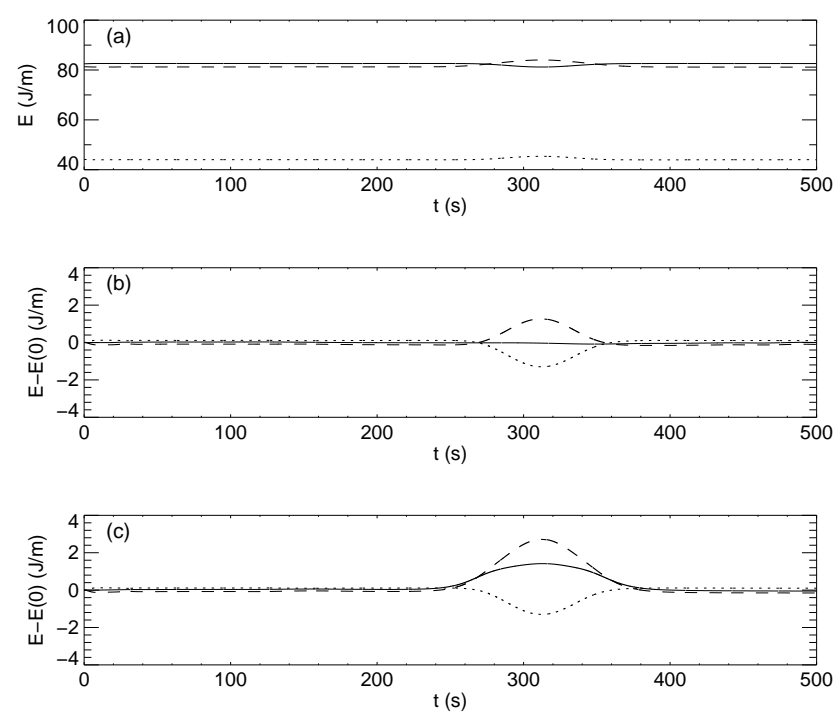

Fig. 5. Energetics for ISW interaction. (a) Solid curve: $\bar{E}_{\text {a }}$. Dots: $\bar{E}_{\mathrm{kp}}$. Dashed: $\bar{E}_{\mathrm{k} 2}$. (b) Dashed: $\bar{E}_{\mathrm{kp}}-\bar{E}_{\mathrm{kp}}(0)$. Dots: $\bar{E}_{\mathrm{a}}-\bar{E}_{\mathrm{a}}(0)$. Solid curve: their sum. (c) Dashed: $\bar{E}_{\mathrm{k} 2}-\bar{E}_{\mathrm{k} 2}(0)$. Dots: $\bar{E}_{\mathrm{a}}-$ $\bar{E}_{\mathrm{a}}(0)$. Solid curve: their sum.

Fig. 5b. During the wave interaction the perturbation kinetic energy rises while the available potential energy drops. They return to their original values after the interaction. The total perturbation energy stays constant during the interaction as 
Table 1. Change in $\bar{E}_{\mathrm{a}}$ and $\bar{E}_{\mathrm{kp}}$ during the interaction of two ISWs. In all cases $\Delta \rho=0.04,\left(z_{\mathrm{pyc}}, d_{\text {pyc }}\right)=(-0.2,0.05) \mathrm{m}$ and $d_{\mathrm{s}}=0.03 \mathrm{~m}$.

\begin{tabular}{cccccccc}
\hline $\begin{array}{c}z_{\mathrm{s}} \\
(\mathrm{m})\end{array}$ & $\begin{array}{c}U_{m} \\
\left(\mathrm{~m} \mathrm{~s}^{-1}\right)\end{array}$ & $\begin{array}{c}\bar{E}_{\mathrm{a} 1} \\
\left(\mathrm{~J} \mathrm{~m}^{-1}\right)\end{array}$ & $\begin{array}{c}\bar{E}_{\mathrm{a} 2} \\
\left(\mathrm{~J} \mathrm{~m}^{-1}\right)\end{array}$ & $\begin{array}{c}\text { \% decrease } \\
\text { in } \bar{E}_{\mathrm{a}}\end{array}$ & $\begin{array}{c}\bar{E}_{\mathrm{kp}}(0) \\
\left(\mathrm{J} \mathrm{m}^{-1}\right)\end{array}$ & $\begin{array}{c}\max \left|\Delta \bar{E}_{\mathrm{kp}}\right| \\
\left(\mathrm{J} \mathrm{m}^{-1}\right)\end{array}$ & $\begin{array}{c}\text { \% increase } \\
\text { in } \bar{E}_{\mathrm{kp}}\end{array}$ \\
\hline-0.3 & 0.0 & 2.5 & 80 & 1.8 & 85.2 & 1.5 & 1.7 \\
- & -0.05 & - & - & 1.8 & 63.0 & 1.5 & 2.3 \\
- & -0.1 & - & - & 1.7 & 43.9 & 1.4 & 3.2 \\
- & - & 5 & - & 1.7 & 44.8 & 1.4 & 3.1 \\
-0.2 & -0.05 & 2.5 & - & 1.7 & 61.8 & 1.4 & 2.3 \\
- & -0.1 & - & - & 1.5 & 42.9 & 1.3 & 2.9 \\
- & -0.15 & - & - & 1.3 & 27.6 & 1.0 & 3.8 \\
- & -0.2 & - & - & 1.0 & 15.2 & 0.8 & 5.3 \\
\hline
\end{tabular}

expected. In Fig. 5c the relative changes of $\bar{E}_{\mathrm{k} 2}$ and $\bar{E}_{\mathrm{a}}$ and their sum is shown. During the interaction $\bar{E}_{\mathrm{k} 2}+\bar{E}_{\mathrm{a}}$ rises by about $1.2 \%$ above its initial value. During the interaction the increase in $\bar{E}_{\mathrm{k} 2}$ is slightly more than twice the increase in $\bar{E}_{\mathrm{kp}}$. Figure 6 shows the energy balance for the secondorder wave energies. Shown is the time rate of change of the $\bar{E}_{\mathrm{k} 2}+\bar{E}_{\mathrm{a}}$ and the negative of the shear production term $P$ along with their sum. Note values have been multiplied by $10^{2}$. The sum is nearly constant but is noisy with fluctuations with amplitude approximately $2.5 \%$ of the variations of the largest term (i.e., with an amplitude of about $10^{-3} \mathrm{~W} \mathrm{~m}^{-1}$ ). This plot illustrates that the $1.2 \%$ rise of $\bar{E}_{\mathrm{k} 2}+\bar{E}_{\mathrm{a}}$ during the interaction is much larger than the numerical errors in the simulation.

Percentage changes of $\bar{E}_{\mathrm{a}}$ and $\bar{E}_{\mathrm{kp}}$ for several cases are given in Table 1. For the simulations that have been done, during the interaction $\bar{E}_{\mathrm{kp}}$ increases by between 2.1 and $3.4 \%$. It increases as $U_{m}$ increases in magnitude. $\bar{E}_{\mathrm{a}}$ decreases by between 1.1 and $1.7 \%$. It decreases as $U_{m}$ increases in magnitude. Only runs with waves of very different amplitudes have been done, otherwise the difference in propagation speeds is very small and the interaction takes a long time.

\subsection{Shoaling ISW wave passing through a turning point}

The second case considered is that of an ISW shoaling through a turning point. The bathymetry has the form

$h(x)=0.25\left(1+\tanh \left(\frac{x}{7.0}\right)\right)$,

the sheared current is the same as in the previous case, and the background density field is given by (41) with $\Delta \rho=0.04$, $\left(z_{\text {pyc }}, d_{\text {pyc }}\right)=(-0.3,0.025) \mathrm{m}$. The pycnocline and shear layer are now at the same depth and the pycnocline is slightly thinner than the shear layer. In the deep/shallow water the shear layer and the pycnocline are in the upper/lower half of the water column, hence ISWs are waves of depression/elevation. As an ISW shoals from deep water it passes through the turning point, where the pycnocline and shear

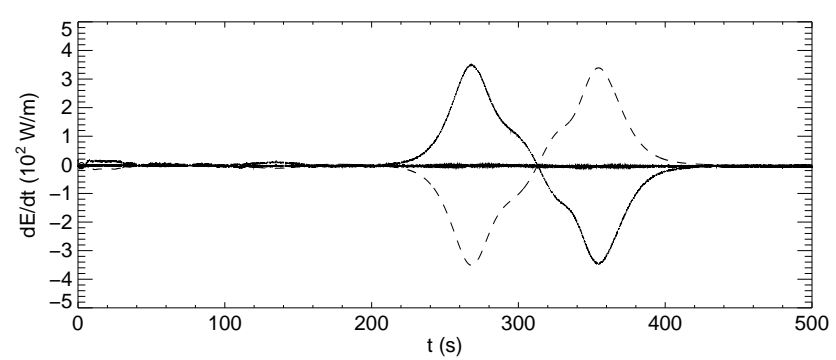

Fig. 6. Second-order energy balance for ISW interaction. Dots: $d\left(\bar{E}_{\mathrm{k} 2}+\bar{E}_{\mathrm{a}}\right) / d t$. Dashed: $P=\iint_{\mathcal{D}} u^{\prime} w \frac{d \bar{U}}{d z}$. Solid curve: their sum.

layer are at mid-depth, and the ISW is transformed into a train of ISWs of elevation riding on a broad depression (Grimshaw et al., 1999) as depicted in Fig. 7 for a wave with initial APE of $\bar{E}_{\mathrm{a}}=10 \mathrm{~J} \mathrm{~m}^{-1}$.

The energy evolution is depicted in Fig. 8. Panel a shows the time evolution of $\bar{E}_{\mathrm{a}}, \bar{E}_{\mathrm{kp}}$ and $\bar{E}_{\mathrm{k} 2}$. The perturbation kinetic energy $\bar{E}_{\mathrm{kp}}$ is initially negative as the addition of the wave decreases the kinetic energy in the system. As the wave shoals both $\bar{E}_{\mathrm{kp}}$ and $\bar{E}_{\mathrm{a}}$ increase. This result can be contrasted with the results of Lamb and Nguyen (2009) who found that for shoaling waves (in this case with the pycnocline intersecting the sloping bottom), as waves shoaled the kinetic energy dropped and the APE rose while the sum was almost constant (it decreased slowly due to viscous losses before dropping rapidly when the waves broke). In the current situation both rise. This can be attributed to a decrease in wave induced currents ( $\bar{E}_{\mathrm{k} 2}$ drops slightly, see panel c) during the early stages of shoaling which in this case results in an increase in kinetic energy.

Figure $8 \mathrm{~b}$ shows the change in $\bar{E}_{\mathrm{a}}$ and $\bar{E}_{\mathrm{kp}}$ from their initial values along with their sum. In panel $c$ the change in the second-order kinetic energy $\bar{E}_{\mathrm{k} 2}$ and $\bar{E}_{\mathrm{a}}$ along with their sum is shown. Both the total perturbation energy and the secondorder perturbation energy rise as the waves shoal. After the waves are on top of the shelf the total perturbation energy 

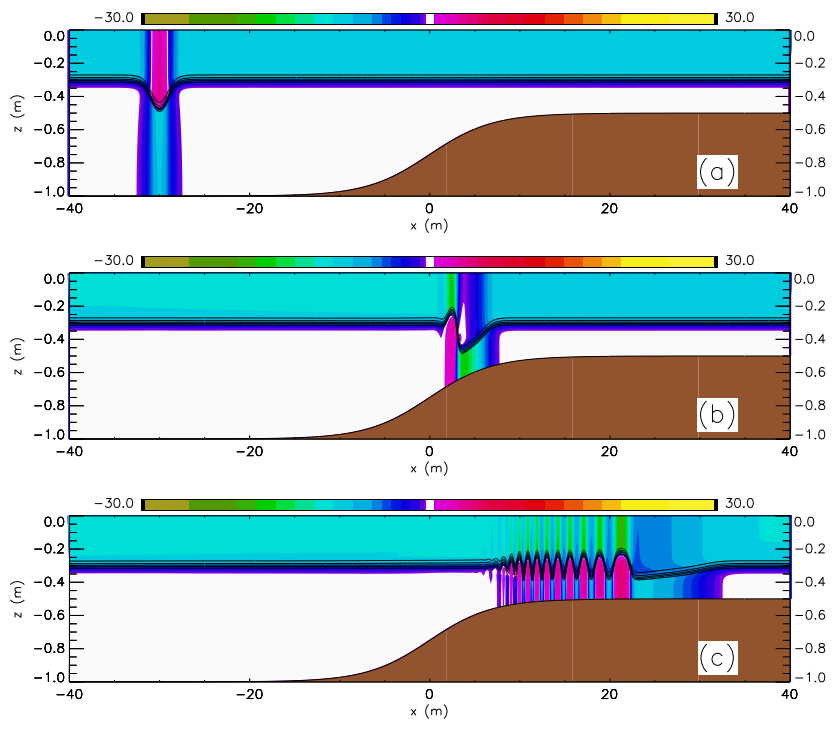

Fig. 7. Contour plots of the density field (solid lines) and horizontal velocity field (colours) for an ISW shoaling through a turning point. (a) Initial wave $(t=0)$. (b) $t=150 \mathrm{~s}$. (c) $t=300 \mathrm{~s}$. The white regions indicate regions where $|u|<0.0013 \mathrm{~m} \mathrm{~s}^{-1}$.
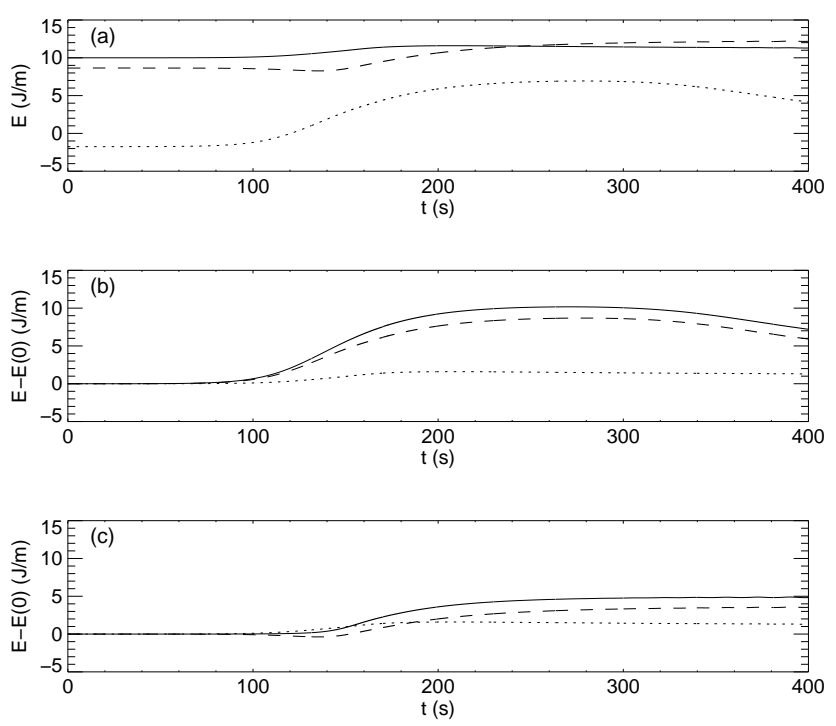

Fig. 8. Energetics for an ISW shoaling through a turning point. (a) Solid curve: $\bar{E}_{\mathrm{a}}$. Dots: $\bar{E}_{\mathrm{kp}}$. Dashed: $\bar{E}_{\mathrm{k} 2}$. (b) Dots: $\bar{E}_{\mathrm{a}}-$ $\bar{E}_{\mathrm{a}}(0)$. Dashed: $\bar{E}_{\mathrm{kp}}-\bar{E}_{\mathrm{kp}}(0)$. Solid curve: their sum. (c) Dots: $\bar{E}_{\mathrm{a}}-\bar{E}_{\mathrm{a}}(0)$. Dashed: $\bar{E}_{\mathrm{k} 2}-\bar{E}_{\mathrm{k} 2}(0)$. Solid curve: their sum.

decreases. The increase in the total perturbation energy is approximately double that of the second-order perturbation energy.

The energy balance is shown in Fig. 9. The top panel compares the terms in the perturbation energy balance equation (30), while the lower panel shows the terms in the secondorder energy balance equation (39). These show that the si-
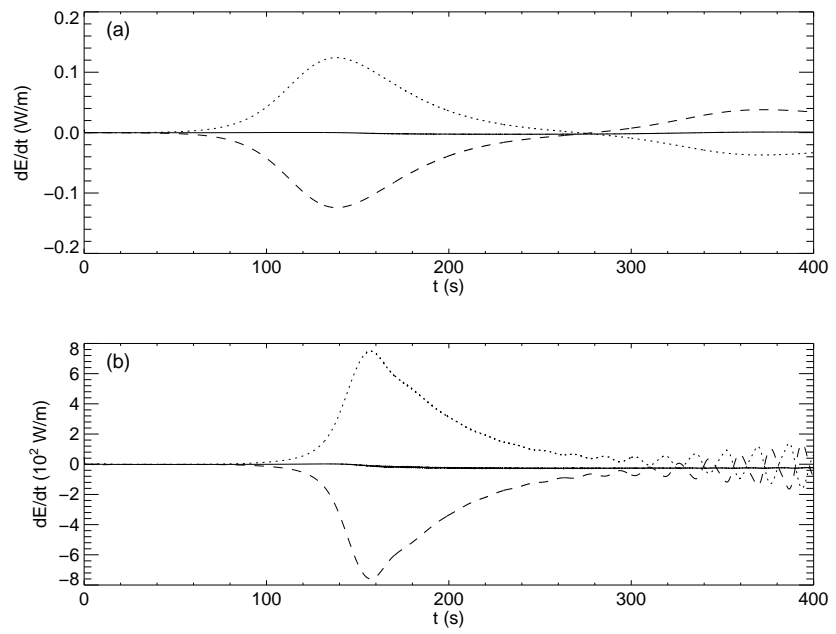

Fig. 9. Energy balance for an ISW shoaling through a turning point (a) Dots: $d\left(\bar{E}_{\mathrm{kp}}+\bar{E}_{\mathrm{a}}\right) / d t$. Dashed: $\Delta p_{\mathrm{s}} M$. Solid: their sum. (b) Dots: $d\left(\bar{E}_{\mathrm{k} 2}+\bar{E}_{\mathrm{a}}\right) / d t$. Dashed: $\iint_{\mathcal{D}} u^{\prime} w \frac{d \bar{U}}{d z}$. Solid: their sum. Note different scales used in each panel.

mulation is conserving energy to high accuracy and that the changes in the total and second-order perturbation energies shown in Fig. 8 are accurate. It also shows the importance of the pressure work and shear production terms in maintaining the energy balance.

Results from several simulations are shown in Figs. 10 and 11. Three runs, using initial available potential energies of 10,20 , and $30 \mathrm{~J} \mathrm{~m}^{-1}$ were done for $U_{m}=-0.1$ and $-0.2 \mathrm{~m} \mathrm{~s}^{-1}$. The simulations using the largest waves were discontinued part-way through because of strong overturning. One run using an initial APE of $10 \mathrm{~J}^{-1}$ for a surface current flowing on to the shelf, with $U_{m}=0.1 \mathrm{~m} \mathrm{~s}^{-1}$, was also done. Figure 10 compares the evolution of $\bar{E}_{\mathrm{a}}, \bar{E}_{\mathrm{kp}}$ and their sum. For the cases with flow off the shelf both the available and perturbation kinetic energies increase initially as the waves shoal. The increase becomes larger as the wave amplitude and strength of the counter-current increase. For the case with an on-shelf current both $\bar{E}_{\mathrm{a}}$ and $\bar{E}_{\mathrm{kp}}$ decrease as the wave shoals. This wave propagates faster than the others and reached the right boundary shortly before the end of the run. This accounts for the slight dip in values starting at about $t=360 \mathrm{~s}$.

Figure 11 shows the relative change in the total energy. For the cases with $U_{m}=-0.1 \mathrm{~m} \mathrm{~s}^{-1}$ the total energy increases by a factor of about 2.2 for the smallest wave and by about 1.5 for the largest wave. For the case with an on-shelf current the total energy decreases by about $50 \%$. For the case with the stronger off-shore currents the total energy increases by a factor of about 35 for the smallest wave. This strong increase occurs because the initial perturbation kinetic energy is negative with almost the same magnitude as the initial available potential energy, making the total initial energy perturbation very small. 

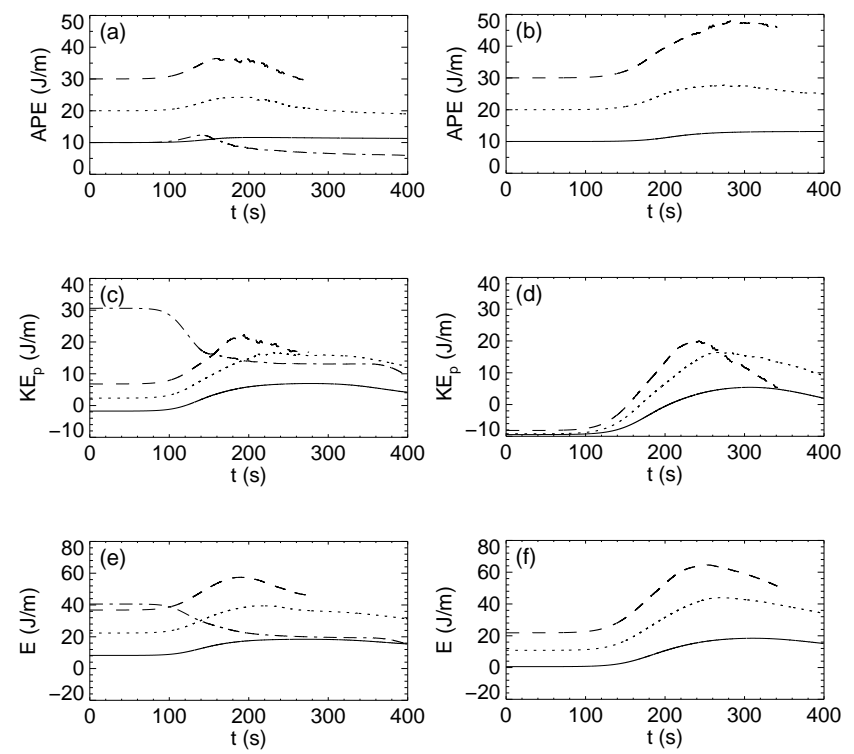

Fig. 10. Evolution of the perturbation energy for simulations of internal solitary waves passing through a turning point. (a, b) Available potential energy $\bar{E}_{\mathrm{a}}$. (c, d) Perturbation kinetic energy $\bar{E}_{\mathrm{kp}}$. (e, f) Total perturbation energy $\bar{E}_{\mathrm{a}}+\bar{E}_{\mathrm{kp}}$. (a, c, e) and (b, d, f) are for $U_{m}=-0.1$ and $-0.2 \mathrm{~m} \mathrm{~s}^{-1}$, respectively. Curves are for different initial wave amplitudes: $\bar{E}_{\mathrm{a}}(0)=10$ (solid), 20 (dots) and 30 (dashed) $\mathrm{J} \mathrm{m}^{-1}$. Dash-dot curve in panels (a, c, e) are for wave with $\bar{E}_{\mathrm{a}}(0)=10 \mathrm{~J} \mathrm{~m}^{-1}$ and $U_{m}=0.1 \mathrm{~m} \mathrm{~s}^{-1}$.

\subsection{ISW reflection}

We next consider the case of an ISW reflecting from a sloping boundary. Because strong wave breaking occurs in this case we solve the Navier-Stokes equations with constant viscosity and diffusivity $\left(10^{-7}\right.$ and $10^{-9} \mathrm{~m}^{2} \mathrm{~s}^{-1}$, respectively) (Lamb and Nguyen, 2009). The choice of these sub-molecular values is explained below. A no-slip bottom boundary condition is used. The bathymetry for this case is given by a smoothed piecewise-linear topography of the form

$h(x)=\frac{S_{\mathrm{s}}}{2}\left(\operatorname{inttanh}\left(x, x_{1}, w\right)-\operatorname{inttanh}\left(x, x_{2}, w\right)\right)$,

where

$$
\begin{aligned}
\operatorname{inttanh}\left(x, x_{0}, w\right) & =\int_{-\infty}^{x}\left(1+\tanh \left(\frac{x^{\prime}-x_{0}}{w}\right)\right) d x^{\prime} \\
& =x-x_{0}+w \ln \left(2 \cosh \left(\frac{x-x_{0}}{w}\right)\right)
\end{aligned}
$$

is a function whose slope varies smoothly from 0 to 2 at $x=x_{0}$ over a length scale $w$. Thus, the slope of the topography $h(x)$ smoothly changes from 0 to $S_{\mathrm{s}}$ at $x=x_{1}$ and then smoothly decreases to zero at $x_{2}$. For this simulation $S_{\mathrm{s}}=0.2, w=0.1 \mathrm{~m}$ and $x_{1}=0$. The value of $x_{2}$ is chosen so that the height of the shelf is $0.85 \mathrm{~m}$. In contrast to previous
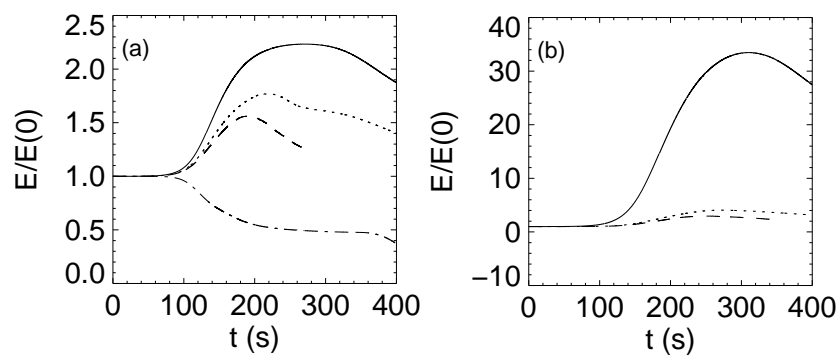

Fig. 11. Evolution of total perturbation energy normalized by the initial energy. Same cases as in Fig. 10. (a) $U_{m}=-0.1 \mathrm{~m} \mathrm{~s}^{-1}$. (b) $U_{m}=-0.2 \mathrm{~m} \mathrm{~s}^{-1}$.
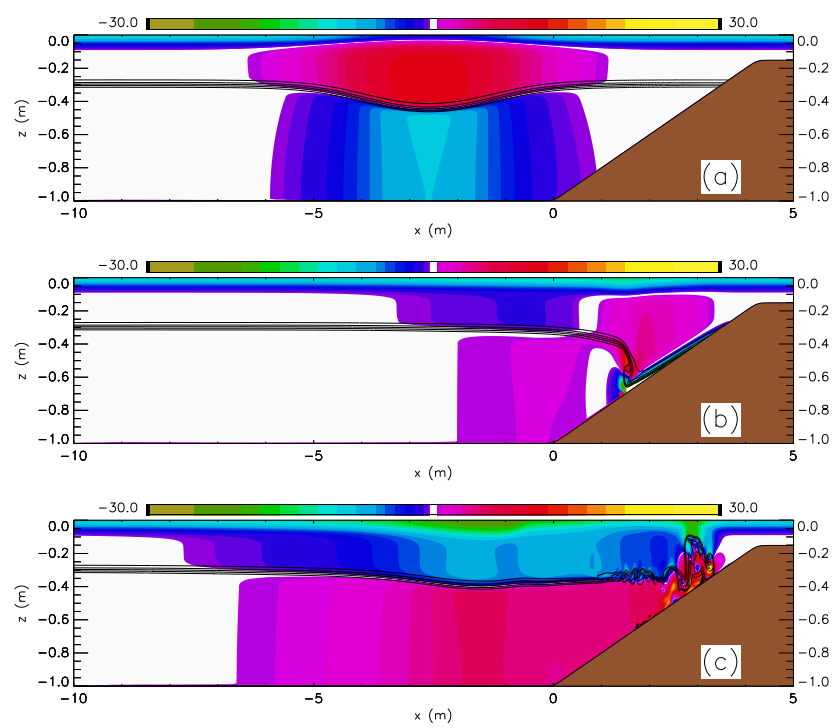

Fig. 12. Contour plots of the density field (solid lines) and horizontal velocity field (colours) for a shoaling ISW with the pycnocline intersecting a sloping bottom boundary. (a) $t=25 \mathrm{~s}$. (b) $t=45 \mathrm{~s}$. (c) $t=60 \mathrm{~s}$. The white regions indicate regions where $|u|<0.0013 \mathrm{~m} \mathrm{~s}^{-1}$.

cases the background current is given by

$\bar{U}(z)=-0.1 e^{-400 z^{2}} \mathrm{~m} \mathrm{~s}^{-1}$,

which has a negligible value of $1.4 \times 10^{-5} \mathrm{~m} \mathrm{~s}^{-1}$ at the top of the shelf. The background density is the same as that used in the shoaling ISW case. The pycnocline now intersects the slope. The initial wave, located at $x=-10 \mathrm{~m}$, has an APE of $10 \mathrm{~J} \mathrm{~m}^{-1}$ and an amplitude of $0.15 \mathrm{~m}$. As the wave shoals it breaks and some of its energy is reflected and the rest is lost to viscous dissipation and mixing. The wave breaking and reflection process is shown in Fig. 12 while the evolution of the wave energies is shown in Fig. 13. As the wave shoals the APE increases while the perturbation kinetic energies (both $\bar{E}_{\mathrm{kp}}$ and $\bar{E}_{\mathrm{k} 2}$ ) decrease, before returning to close to their initial values. The total perturbation energy increases during the breaking/reflection process after which it decreases slightly 

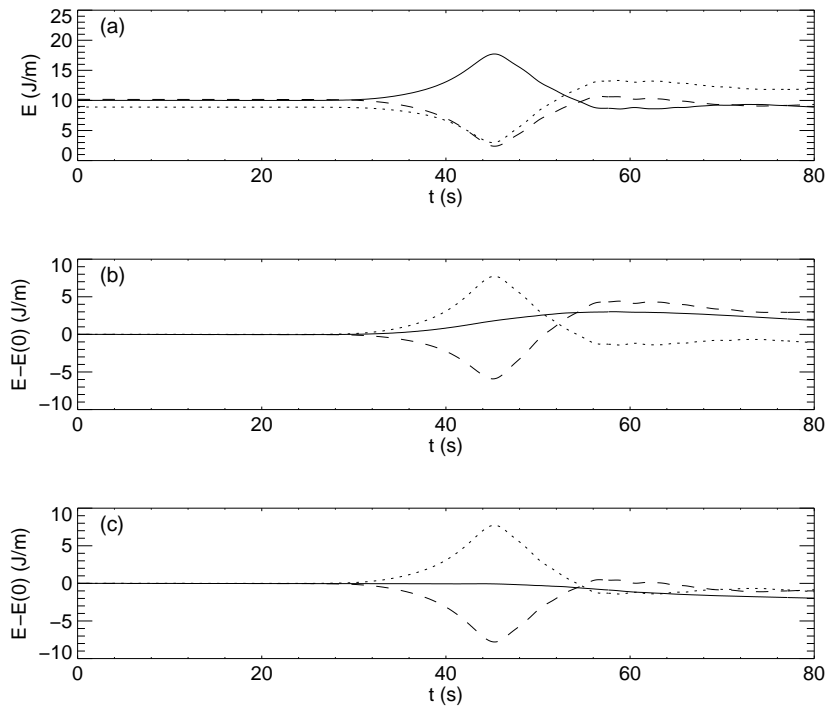

Fig. 13. Energetics for a shoaling ISW undergoing reflection. (a) Solid curve: $\bar{E}_{\mathrm{a}}$. Dots: $\bar{E}_{\mathrm{kp}}$. Dashed: $\bar{E}_{\mathrm{k} 2}$. (b) Dots: $\bar{E}_{\mathrm{a}}-\bar{E}_{\mathrm{a}}(0)$. Dashed: $\bar{E}_{\mathrm{kp}}-\bar{E}_{\mathrm{kp}}(0)$. Solid curve: their sum. (c) Dots: $\bar{E}_{\mathrm{a}}-\bar{E}_{\mathrm{a}}(0)$. Dashed: $\bar{E}_{\mathrm{k} 2}-\bar{E}_{\mathrm{k} 2}(0)$. Solid curve: their sum.

(Fig. 13b). At the end of the simulation it is about $10 \%$ larger than it was initially. The small values of the viscosity and diffusivity were used to illustrate the fact that the total perturbation energy can increase after reflection in spite of the mixing and dissipation associated with the wave breaking process. In contrast, the second-order perturbation energy decreases monotonically (panel c).

\subsection{A deep water internal seiche}

The final case considered is that of a deep water internal seiche. The symmetric bathymetry consists of two hyperbolictangent shelves at either end of the domain of the form

$h(x)=\frac{a}{2}\left(2+\tanh \left(\frac{x+x_{0}}{d}\right)-\tanh \left(\frac{x-x_{0}}{d}\right)\right)$,

with shelf amplitudes $a=0.5 \mathrm{~m}$ and with $x_{0}=15 \mathrm{~m}$ and $d=2.0 \mathrm{~m}$. The left and right boundaries are at $x= \pm 30 \mathrm{~m}$. The initial stratification stratification consists of a sloping pycnocline specified by

$\rho(x, z, 0)=\bar{\rho}(z-\alpha x)$

where $\bar{\rho}$ is given by (41) with $\Delta \rho=0.04, z_{\text {pyc }}=-0.8 \mathrm{~m}$ and $d_{\text {pyc }}=0.025 \mathrm{~m}$. The initial slope of the pycnocline is $\alpha=$ -0.005 . Above the shelves a surface trapped current of the form (42) is added using $U_{m}=-0.1 \mathrm{~m} \mathrm{~s}^{-1}, z_{\mathrm{s}}=-0.2 \mathrm{~m}$ and $d_{\mathrm{s}}=0.05 \mathrm{~m} . \bar{U}$ is effectively zero at the top of the shelves.

Figure 14 shows the density field and velocity fields at $t=0$ and at two later times. At $t=60 \mathrm{~s}$, approximately a
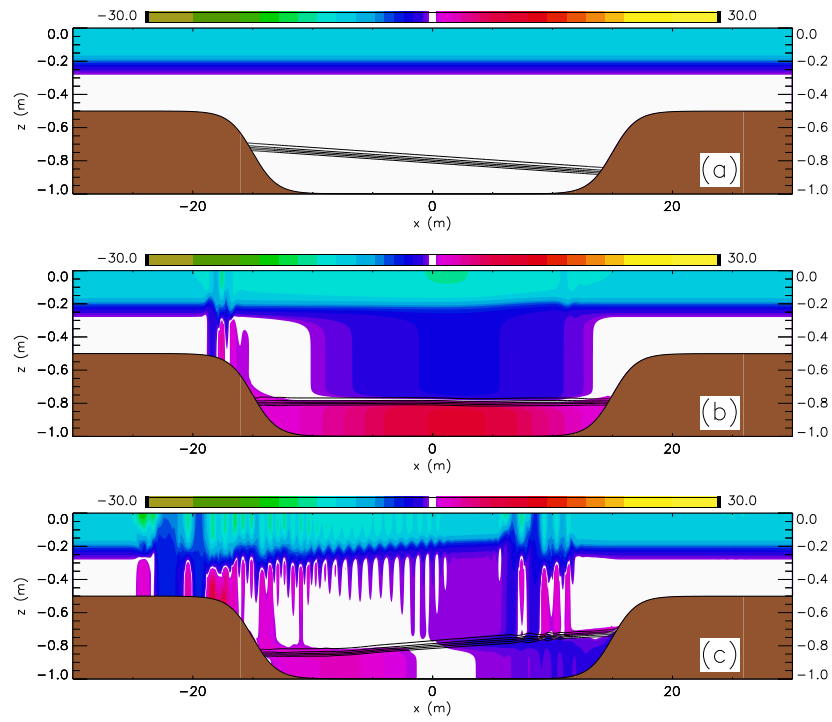

Fig. 14. Contour plots of the density field (solid lines) and horizontal velocity field (colours) for the deep seiche. (a) Initial state $(t=0)$. (b) $t=60 \mathrm{~s}$. (c) $t=120 \mathrm{~s}$. The white regions indicate regions where $|u|<0.0013 \mathrm{~m} \mathrm{~s}^{-1}$.

quarter of an internal seiche period, the pycnocline is horizontal. Associated with the relaxation of the pycnocline are rightward/leftward currents beneath/above the pycnocline. A shear wave can be seen propagating leftward onto the shelf. At $t=120 \mathrm{~s}$ the pycnocline is close to its maximum positive slope. Shear instabilities have formed in the shear layer above.

To calculate the APE we use $\bar{\rho}(z)$ as the reference density. If the shelf was given by step like topography (given by $d \rightarrow 0$ ), this would be the sorted density field however because of the sloping sidewalls of the basin the sorted density field will be slightly different. Thus there is a small time independent error in our calculation of the APE however we are only interested in variations in the APE which are unaffected by our choice of the reference density.

When the sloping pycnocline is released the internal seiche, confined to the basin, evolves as the initial APE is converted to KE. Figure 15 shows the evolution of the internal seiche energy. In panel (a) the perturbation kinetic energy $\bar{E}_{\mathrm{kp}}$, the APE $\bar{E}_{\mathrm{a}}$ and their sum are shown. Since $u^{\prime}=0$ initially $\bar{E}_{\mathrm{kp}}(0)=0$. It rises while $\bar{E}_{\mathrm{a}}$ falls as APE is converted to kinetic energy. The total perturbation energy $\bar{E}_{\mathrm{p}}=\bar{E}_{\mathrm{kp}}+\bar{E}_{\mathrm{a}}$ is not constant, rising by about $70 \%$ after a quarter of a seiche period after which it falls until the end of the run (at approximately 5/8 of a seiche period). Panel (b) shows the time evolution of $\bar{E}_{\mathrm{k} 2}, \bar{E}_{\mathrm{a}}$ and their sum. The total second-order energy perturbation is almost constant over the first $60 \mathrm{~s}$ after which it rises. It has doubled by the end of the run. In panel (c) we verify the energy balance equation (30). This shows that the change in the total energy seen 


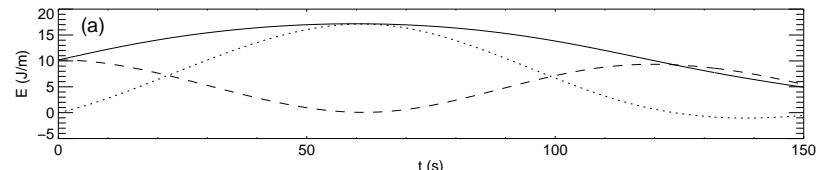

$t(s)$

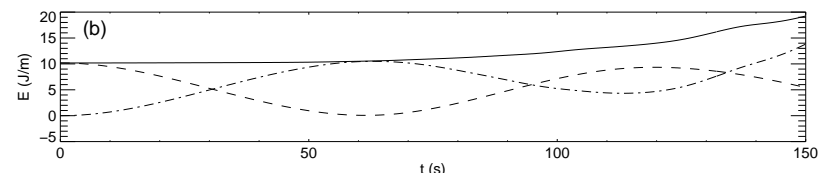

$t(s)$

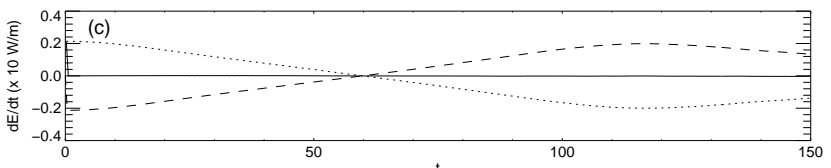

Fig. 15. Energetics for deep seiche case. (a) Dots: $\bar{E}_{\mathrm{kp}}$. Dashed: $\bar{E}_{\mathrm{a}}$. Solid: their sum. (b) Dots: $\bar{E}_{\mathrm{k} 2}$. Dashed: $\bar{E}_{\mathrm{a}}$. Solid: their sum. (c) Dots: $d\left(\bar{E}_{\mathrm{kp}}+\bar{E}_{\mathrm{a}}\right) / d t$. Dashed: $\Delta p_{\mathrm{s}} M$. Solid: their sum.

in panel (a) is accounted for by the pressure drop across the domain which results in fluid outside the domain doing net work on the fluid in the interior.

Results for a case with the surface current in the opposite direction are shown in Fig. 16. The perturbation kinetic energy decreases initially and becomes negative as the induced currents act to reduce the horizontal velocity in the surface current. In contrast, the second-order energy rises monotonically as in the previous case.

\section{Mechanism for the change in total perturbation energy}

In the above cases that did not include viscosity we have seen that the change in the total perturbation energy can be accounted for by the net horizontal pressure gradient that forms across the domain. This is a consequence of the boundary conditions used in the model. Consider the deep seiche. When the pycnocline is released the fluid beneath it flows rightward as a consequence of a negative pressure gradient. As the left half of the pycnocline drops and the right half rises the fluid above the pycnocline will be accelerated to the left. This requires a positive horizontal pressure gradient, i.e., the pressure is higher above the right half of the pycnocline than it is over the left half. On the shelves the boundary conditions used in the model $\left(u_{t}=0\right)$ prohibit the fluid from accelerating. Thus the pressure is horizontally uniform on the shelves. The result is that the pressure is higher at the right boundary that it is at the left boundary. Because the fluid enters the domain at the right boundary and leaves through the left boundary ( $\bar{U}$ is negative) the work done by the fluid outside the domain, during the early stages of the flow evolution, acts to increase the energy in the system. This continues

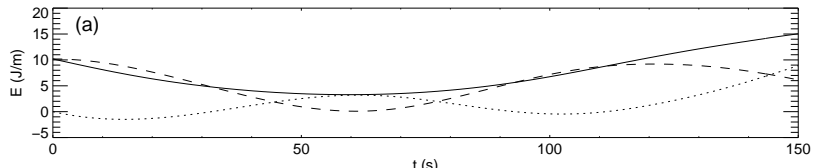

$\mathrm{t}(\mathrm{s})$
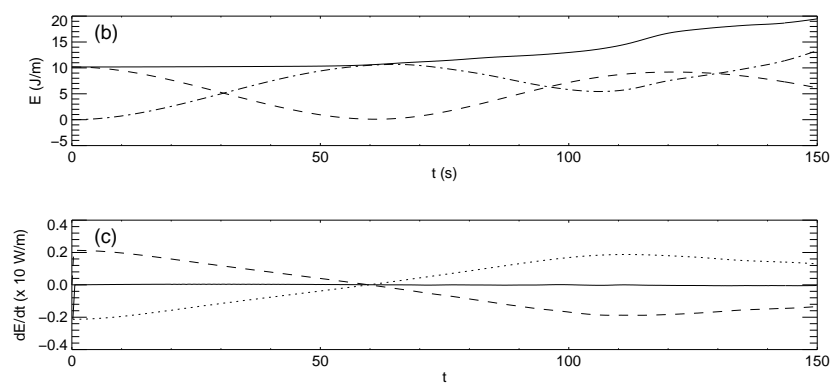

Fig. 16. Same as Fig. 15 except that surface current is in opposite direction.

until the pynocline levels out at about $t=60 \mathrm{~s}$. Fluid above the pycnocline is now accelerated to the right. The pressure drop across the basin reverses sign and the pressure exerted by fluid outside the domain acts to decrease the energy in the system.

It is clear that this behaviour is associated with the choice of boundary conditions. One can imagine constructing a periodic series of shelves and basins, arranged in an annulus, with identically sloping pycnoclines in each basin. When released from rest the flow would be somewhat different because the pressure perturbation would have to be periodic. Now as the pressure rises/falls above the depressed/elevated edges of the pycnocline a rightward flow would be forced across the tops of the shelves. Associated with the acceleration of this rightward flow the pressure perturbation would decrease across the tops of the shelves in such a way that the pressure perturbation remains zero at the midpoints of the shelves.

The use of a rigid lid is an essential ingredient in the results of these numerical simulations because it is responsible for forcing the pressure field to remain constant on the top of the shelves. If there was a free surface this would not be possible. In the deep seiche case we can anticipate that surface waves would be generated above the edges of the sloping pycnocline beyond which the pressure field would be unperturbed. This is verified in the next section.

\section{The deep internal seiche with a free surface}

To further explore the mechanism behind the change in total perturbation energy we now modify the deep internal seiche case by adding a free surface. This is done by adding a layer of air $0.5 \mathrm{~m}$ thick above the water column and solving the non-Boussinesq equations. The background density field 


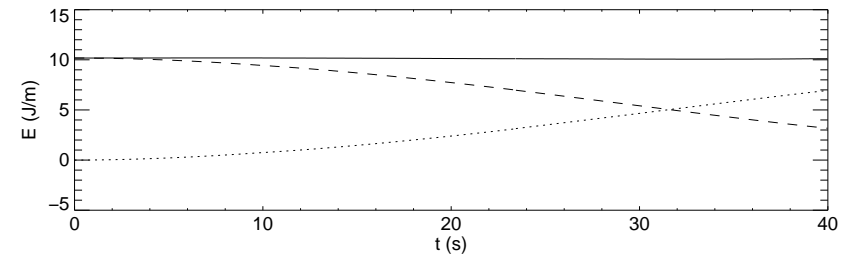

Fig. 17. Energetics for deep seiche case with a free surface. Dots: $\bar{E}_{\mathrm{kp}}$. Dashed: $\bar{E}_{\mathrm{a}}$. Solid: their sum.

consists of a layer of air with density $1 \mathrm{~kg} \mathrm{~m}^{-3}$, a fresh water surface layer of density $1000 \mathrm{~kg} \mathrm{~m}^{-3}$ and a bottom layer of density $1040 \mathrm{~kg} \mathrm{~m}^{-3}$. A sharp interface has not been incorporated into the model so the "free" surface is a diffuse surface of finite thickness. The background density is given by

$$
\begin{aligned}
& \rho_{0}(1+\bar{\rho}(z))=1040-20\left(1+\tanh \left(\frac{z-z_{\mathrm{pyc}}}{d_{\mathrm{pyc}}}\right)\right) \\
& -\frac{999}{2}\left(1+\tanh \left(\frac{z-z_{\text {surf }}}{d_{\text {surf }}}\right)\right) \mathrm{kg} \mathrm{m}^{-3} .
\end{aligned}
$$

The free surface is at $z_{\text {surf }}=0$ with a thickness $d_{\text {surf }}=$ $0.002 \mathrm{~m}$. The pycnocline depth and thickness is the same as in the Boussinesq version of the deep seiche. The same background velocity field is also used with the current extending up above the free surface.

The non-Boussinesq simulation with a free surface is very computationally demanding for several reasons. First the projection operator must be calculated at each time step which increases the run time by a factor of 4-8 per time step (depending on resolution). In addition a much higher vertical resolution was required to resolve the thin free surface. There is also a time step restriction associated with wave propagation speeds. The use of a free surface introduces surface waves into the system and a concomitant reduction in the time step is necessary. The presence of surface waves also results in the requirement for a longer domain. Hence only one simulation has been done and it was run for $40 \mathrm{~s}$.

Figure 17 shows the time evolution of $\bar{E}_{\mathrm{kp}}, \bar{E}_{\mathrm{a}}$ and their sum. The total energy remains nearly constant (it decreases by less than $1.2 \%$ ). In comparison, in the Boussinesq case with a rigid water surface the total perturbation energy had risen by about $50 \%$ in the same period of time. The fact that the total energy is constant in this case is a consequence of the pressure rise across the domain being reduced by three orders of magnitude.

Figure 18 compares the horizontal distribution of the vertically integrated kinetic energy perturbation for the deep internal seiche cases. The Boussinesq case discussed above is obtained from the non-Boussinesq case by using $\rho_{0}=1000 \mathrm{~kg} \mathrm{~m}^{-3}$ as the reference density. An additional run using $\rho_{0}=1020 \mathrm{~kg} \mathrm{~m}^{-3}$ as the reference density (giving $\Delta \rho=0.0392$ ) was done to test the sensitivity to the choice
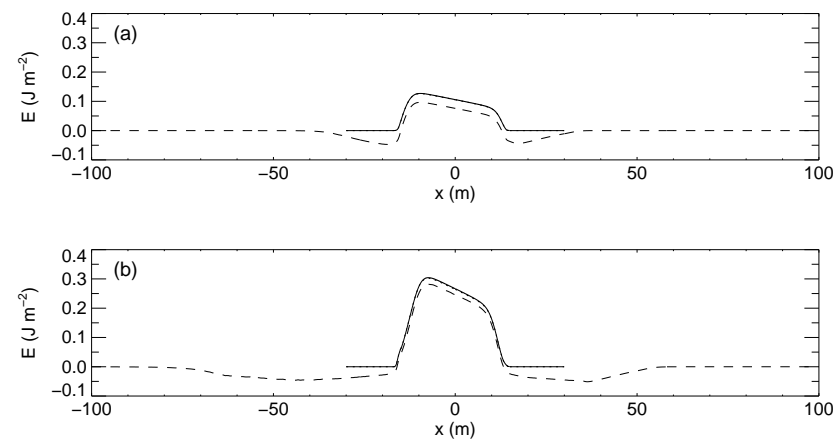

Fig. 18. Vertically integrated kinetic energy perturbation for the deep seiche cases: comparison of Boussinesq and non-Boussinesq simulations. Solid line: Boussinesq (domain between $\pm 30 \mathrm{~m}$ ) using $\rho_{0}=1000 \mathrm{~kg} \mathrm{~m}^{-3}$. Dots: Boussinesq using $\rho_{0}=1020 \mathrm{~kg} \mathrm{~m}^{-3}$ (virtually indistinguishable from solid curve). Dashed line: nonBoussinesq. (a) $t=10 \mathrm{~s}$. (b) $t=20 \mathrm{~s}$.

of reference density. The results from the two Boussinesq simulations are indistinguishable in Fig. 18. In the nonBoussinesq case with a free surface the kinetic energy above the basin is lower than in the Boussinesq simulations. In addition there are negative values at the two sides of the basin. This is due to the presence of surface waves generated above the edges of the pycnocline. At the right side the free surface is pushed up and a rightward propagating surface wave of elevation is generated. The currents induced by this wave are negative above the free surface and positive below it. The result is that the horizontal velocity in the surface current below the free surface is reduced with a concomitant reduction in the kinetic energy since the kinetic energy perturbation in the overlying air is negligible. At the other end of the basin the downwelling pycnocline results in the generation of a leftward propagating surface wave of depression. The associated induced currents are again positive below the free surface and the kinetic energy perturbation is again negative. By $t=20 \mathrm{~s}$ (panel b) these surface waves have propagated further onto the shelves on either side of the basin with the leftward propagating wave having a faster propagation speed.

An important question is how energy is split between the surface waves and the internal waves. The energy in the surface waves is dominated by the kinetic energy perturbation, it being first-order in the wave amplitude while the potential energy in the wave is second-order, which is negative. This is illustrated in Fig. 19 which compares the total vertically integrated energy perturbation for the Boussinesq and nonBoussinesq simulations at $t=20$ and $40 \mathrm{~s}$. On the shelves the perturbation energy in the non-Boussinesq simulation is negative. Above the basin the energy is similar in the two cases, being slightly smaller in the non-Boussinesq simulation. Both cases agree in some of the fine details, such as the feature between $x=-18$ and $-16 \mathrm{~m}$ at $t=40 \mathrm{~s}$. In the nonBoussinesq simulation an increase in total energy above the basin is compensated for by a negative energy perturbation 

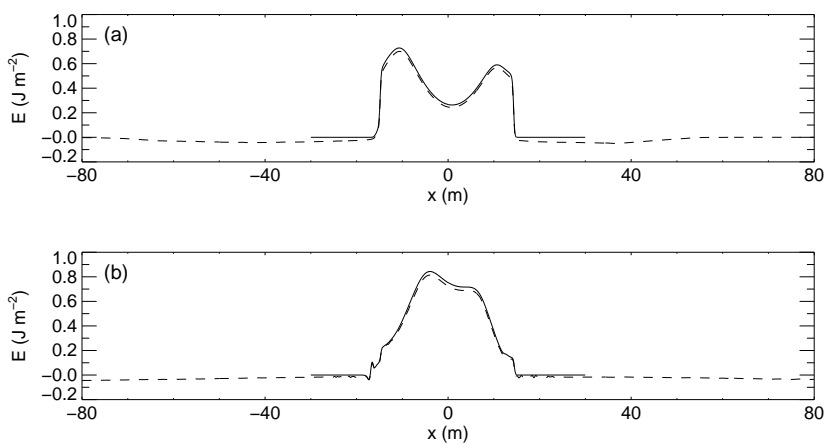

Fig. 19. Vertically integrated energy perturbation, $E_{\mathrm{kp}}+E_{\mathrm{a}}$, for the deep seiche cases: comparison of Boussinesq and non-Boussinesq simulations. Solid line: Boussinesq (domain between $\pm 30 \mathrm{~m}$ ) using $\rho_{0}=1000 \mathrm{~kg} \mathrm{~m}^{-3}$. Dashed line: non-Boussinesq. (a) $t=20 \mathrm{~s}$. (b) $t=40 \mathrm{~s}$.

associated with surface waves. The similarity of the energies above the basin in the two simulations suggests that the change in total perturbation energy in the Boussinesq simulation may be a useful approximation of the change in the baroclinic wave field in the non-Boussinesq case, however this does not take into account the fact that some barotropic energy must be present over the basin in the non-Boussinesq simulation. This needs further investigation.

\section{Conclusions}

The implications of a background sheared current on the evolution of the mechanical energy in four physical situations based on oceanographic conditions have been explored. Three of these involve internal solitary waves, the fourth case being the evolution of an internal seiche trapped in a deep basin. Evolution equations for the total perturbation energy density $E_{\mathrm{kp}}+E_{\mathrm{a}}$ and the second-order perturbation energy density $E_{\mathrm{k} 2}+E_{\mathrm{a}}$ were derived. An appropriate average of the latter is often referred to as the "wave energy". This is appropriate in the context of slowly varying wave trains in which case the background flow $\bar{U}$ can be defined by averaging over the wave train. In that case the averages of $E_{\mathrm{kp}}$ and $E_{\mathrm{k} 2}$ are identical. In the context of ISWs this procedure does not make sense and the first-order kinetic energy perturbation term $\bar{U} u^{\prime}$ plays an important role in the evolution of the mechanical energy of the system and must be included (see also Fabrikant and Stepanyants, 1998). This term makes it possible for the addition of an ISW to reduce the mechanical energy of the system.

The presence of a background sheared current has important consequences for the evolution of the mechanical energy in the system. The domain integrated perturbation energy $\bar{E}_{\mathrm{kp}}+\bar{E}_{\mathrm{a}}$ is conserved however work done on the flow domain by a net horizontal pressure change across the domain can result in significant changes in the perturbation energy if the water depth is not constant. This occurred in all three cases in which the water depth varied and which used a rigid lid at the water surface. In the case of a flat bottomed domain it was shown that the net pressure change must remain zero and hence the total perturbation energy remains constant. In contrast the second-order perturbation energy $\bar{E}_{\mathrm{k} 2}+\bar{E}_{\mathrm{a}}$ need not remain constant. For the case of an ISW reflecting off a sloping boundary it was demonstrated that it is possible for the total mechanical energy to rise after wave breaking occured in spite of energy loss due to dissipation.

It was argued that the change in mechanical energy was a consequence of the lateral boundary conditions which constrained the inflow/outflow fluid velocity to be constant, the use of a rigid lid, and variable water depth which, in combination, can support a pressure change across the domain. To test this the deep internal seiche case was repeated by dropping the Boussinesq approximation and adding a layer of air above the water column, the air and water being separated by a diffuse free surface. In this simulation the pressure drop across the domain was reduced by three orders of magnitude and mechanical energy was almost constant (to within 1\%). Surface waves were generated which carried energy away from the basin containing the deep seiche. It appears that the absence of these waves in the rigid lid, Boussinesq simulation may account for the increase in energy in that case and that the change in total energy in the Boussinesq simulations may accurately predict changes in baroclinic wave energy.

The results highlight the need to investigate the role of a free surface in the context of shoaling ISWs. This will be the subject of future research.

Acknowledgements. This work is supported by a grant from the Natural Sciences and Engineering Research Council of Canada.

Edited by: A. Scotti

Reviewed by: B. White and another anonymous referee

\section{References}

Bell, J. B. and Marcus, D. J.: A second-order projection method for variable-density flows, J. Comput. Phys., 101, 334-348, 1992.

Bell, J. B., Colella, P., and Glaz, H. M.: A second order projection method for the incompressible Navier-Stokes equations, J. Comput. Phys., 85, 257-283, 1989.

Benney, D. J.: Long nonlinear waves in fluid flows, J. Math. Phys., 45, 52-63, 1966.

Bourgault, D., Blokhina, M. D., Mirshak, R., and Kelley, D. E.: Evolution of a shoaling internal solitary wavetrain, Geophys. Res. Lett., 34, L03601, doi:10.1029/2006GL028462, 2007.

Bretherton, F. P.: On the mean motion induced by internal gravity waves, J. Fluid Mech., 36, 785-803, 1969.

Bretherton, F. P. and Garrett, C. J. R.: The propagation of wave trains in moving media, Proc. R. Soc. Lon. Ser.-A, 302, 529-545, 1968.

Carter, G. S., Gregg, M. C., and Lien, R.-C.: Internal waves, solitary-like waves, and mixing on the Monterey Bay shelf, Cont. Shelf Res., 25, 1499-1520, 2005. 
Craik, A. D. D.: Wave interactions and fluid flows, Cambridge University Press, Cambridge, UK, 1985.

Fabrikant, A. and Stepanyants, Y. A.: Propagation of waves in shear flows, World Scientific, 1998.

Gear, J. A. and Grimshaw, R.: A second-order theory for solitary waves in shallow fluids, Phys. Fluids, 26, 14-29, 1983.

Grimshaw, R.: Wave action and wave-mean flow interaction, with application to stratified shear flows, Annu. Rev. Fluid Mech., 16, 11-44, 1985.

Grimshaw, R., Pelinovsky, E., and Talipova, T.: Solitary wave transformation in a medium with sign-variable quadratic nonlinear and cubic nonlinearity, Physica D, 132, 40-62, 1999.

Grimshaw, R., Pelinovsky, E., and Poloukhina, O.: Higher-order Korteweg-de Vries models for internal solitary waves in a stratified shear flow with a free surface, Nonlin. Processes Geophys., 9, 221-235, doi:10.5194/npg-9-221-2002, 2002.

Helfrich, K. R.: Internal solitary wave breaking and run-up on a uniform slope, J. Fluid Mech., 243, 133-154, 1992.

Jeans, D. R. G. and Sherwin, T. J.: The evolution and energetics of large amplitude nonlinear internal waves on the Portuguese shelf, J. Mar. Res., 59, 327-353, 2001.

Klymak, J. M., Pinkel, R., Liu, C.-T., Liu, A. K., and David, L.: Prototypical solitons in the South China Sea, Geophys. Res. Lett., 33, L11607, doi:10.1029/2006GL025 932, 2006.

Lamb, K. G.: Numerical experiments of internal wave generation by strong tidal flow across a finite amplitude bank edge, J. Geophys. Res., 99, 843-864, 1994.

Lamb, K. G.: Conjugate flows for a three-layer fluid, Phys. Fluids, 12, 2169-2185, 2000.

Lamb, K. G.: Shoaling solitary internal waves: on a criterion for the formation of waves with trapped cores, J. Fluid Mech., 478, 81-100, doi:10.1017/S0022112002003269, 2003.

Lamb, K. G.: Energy and pseudoenergy flux in the internal wave field generated by tidal flow over topography, Cont. Shelf Res., 27, 1208-1232, doi:10.1016/j.csr.2007.01.020, 2007.
Lamb, K. G.: On the Calculation of the Available Potential Energy of an Isolated Perturbation in a Density Stratified Fluid, J. Fluid Mech., 597, 415-427, doi:10.1017/S0022112007009743, 2008.

Lamb, K. G. and Nguyen, V. T.: Calculating Energy Flux in Internal Solitary Waves with an Application to Reflectance, J. Phys. Oceanogr., 39, 559-580, doi:10.1175/2008JPO3882.1, 2009.

Lamb, K. G. and Wan, B.: Conjugate flows and flat solitary waves for a continuously stratified fluid, Phys. Fluids, 10, 2061-2079, 1998.

Michallet, H. and Ivey, G. N.: Experiments on mixing due to internal solitary waves breaking on uniform slopes, J. Geophys. Res., 104, 13467-13477, 1999.

Moum, J. N., Klymak, J. M., Nash, J. D., Perlin, A., and Smyth, W. D.: Energy transport by nonlinear internal waves, J. Phys. Oceanogr., 37, 1968-1988, doi:10.1175/JPO3094.1, 2007.

Sandstrom, H. and Elliott, J. A.: Internal tide and solitons on the Scotian Shelf: A nutrient pump at work, J. Geophys. Res., 89, 6415-6426, 1984.

Scotti, A., Beardsley, R., and Butman, B.: On the interpretation of energy and energy fluxes of nonlinear internal waves: an example from Massachusetts Bay, J. Fluid Mech., 561, 103-112, 2006.

Stastna, M. and Lamb, K. G.: Large fully nonlinear internal solitary waves: the effect of background current, Phys. Fluids, 14, 29872999, doi:10.1063/1.1496510, 2002.

Tung, K.-K., Chan, T. F., and Kubota, T.: Large amplitude internal waves of permanent form, Stud. Appl. Math., 66, 1-44, 1982.

Turkington, B., Eydeland, A., and Wang, S.: A computational method for solitary internal waves in a continuously stratified fluid, Stud. Appl. Math., 85, 93-127, 1991.

Turner, R. E. L. and Vanden-Broeck, J. M.: Broadening of interfacial solitary waves, Phys. Fluids, 31, 2486-2490, 1988.

Whitham, G. B.: Linear and Nonlinear Waves, John Wiley \& Sons, USA, 1974.

Zhou, X. and Grimshaw, R.: The effect of variable currents on internal solitary waves, Dynam. Atmos. Oceans, 14, 17-39, 1989. 